\title{
Quantum Lorentz Degrees of Polynomials and a Pólya Theorem for Polynomials Positive on $q$-Lattices
}

\author{
Rachid Ait-Haddou ${ }^{\mathrm{a}}$, Ron Goldman ${ }^{\mathrm{b}}$, Marie-Laurence Mazure ${ }^{\mathrm{c}}$ \\ ${ }^{a}$ Department of Mathematics and Statistics, King Fahd University of Petroleum \& Minerals, Dhahran \\ 31261, Saudi Arabia \\ ${ }^{b}$ Department of Computer Science, Rice University Houston, Texas 77251 USA \\ ${ }^{c}$ Laboratoire Jean Kuntzmann, CNRS, Université Grenoble Alpes, 38000 Grenoble, France
}

\begin{abstract}
We establish the uniform convergence of the control polygons generated by repeated degree elevation of $q$-Bézier curves (i.e., polynomial curves represented in the $q$ Bernstein bases of increasing degrees) on $[0,1], q>1$, to a piecewise linear curve with vertices on the original curve. A similar result is proved for $q<1$, but surprisingly the limit vertices are not on the original curve, but on the $q^{-1}$-Bézier curve with control polygon taken in the reverse order. We introduce a $q$-deformation (quantum Lorentz degree) of the classical notion of Lorentz degree for polynomials and we study its properties. As an application of our convergence results, we introduce a notion of $q$-positivity which guarantees that the $q$-Lorentz degree is finite. We also obtain upper bounds for the quantum Lorentz degrees. Finally, as a by-product we provide a generalization to polynomials positive on $q$-lattices of the univariate Pólya theorem concerning polynomials positive on the non-negative axis.
\end{abstract}

Keywords: quantum Lorentz degrees; quantum Pólya's theorems; $q$-lattices; $q$-Bernstein basis; $q$-positivity; $q$-blossom; degree elevation 2000 MSC: 65D17, 68U05, 26C99, 05A30, 41A99

\section{Introduction}

In 1915 Bernstein observed that a real polynomial $P$ having no zeros in an interval $[a, b]$ admits a representation of the form

$$
P(x)=\sum_{k=0}^{N} a_{k}(x-a)^{k}(b-x)^{N-k}
$$

with all $a_{k}$ non-negative or all $a_{k}$ non-positive [5]. The smallest integer $N$ ensuring such a representation is called the Lorentz degree of the polynomial $P$ on the interval $[a, b]$ and is denoted by $L_{[a, b]}(P)$. The Lorentz degree of polynomials has been the subject of many studies $[1,9,10,11]$. Several improvements of Markov inequalities for polynomials or their derivatives with moderate Lorentz degrees are given in [30, 12].

Bernstein's theorem can be interpreted in terms of degree elevation of Bézier curves in the following sense: A polynomial $P$ of degree at most $n$ with Bézier coefficients

Email addresses: rachid. aithaddou@kfupm.edu.sa (Rachid Ait-Haddou), rng@rice.edu (Ron Goldman), marielaurence.mazure@univ-grenoble-alpes.fr (Marie-Laurence Mazure) 
$p_{0}, p_{1}, \ldots, p_{n}$ relative to $(a, b)$ can also be viewed as a polynomial of degree at most $n+1$ with new Bézier coefficients $r_{0}, r_{1}, \ldots, r_{N+1}$, i.e.,

$$
P(x)=\sum_{k=0}^{n} p_{k} B_{k}^{n}(x)=\sum_{k=0}^{n+1} r_{k} B_{k}^{n+1}(x), \quad x \in[a, b],
$$

where the Bernstein basis is defined by

$$
B_{k}^{n}(x)=\left(\begin{array}{l}
n \\
k
\end{array}\right) \frac{(x-a)^{k}(b-x)^{n-k}}{(b-a)^{n}}, \quad k=0,1, \ldots, n,
$$

and where $r_{0}=p_{0}, r_{n+1}=p_{n}$ and

$$
r_{i}=\frac{n+1-i}{n+1} p_{i}+\frac{i}{n+1} p_{i-1}, i=1, \ldots, n .
$$

The process of iterating formula (3) to express a given polynomial $P$ in Bernstein bases of higher degrees is called degree elevation. It is well known that the piecewise linear interpolant to the Bézier coefficients generated by degree elevation converges uniformly to $P$ on $[a, b]$ [29]. Thus, in particular, if $P$ is positive on $[a, b]$, after sufficiently many degree elevation steps, the Bézier coefficients are all non-negative. This observation coincides with Bernstein's theorem.

It has been shown, for instance in [3], that Bernstein's theorem is equivalent to the celebrated univariate Pólya theorem on positive polynomials [26]. This theorem states that for any polynomial $P$ positive on the interval $[0, \infty[$, there exists an integer $N$ such that the coefficients in the monomial basis of the polynomial $(1+x)^{N} P(x)$ are nonnegative. An equivalence between the multivariate Pólya theorem and a generalization of Bernstein's theorem to multivariate Bézier patches is established in [8, 21].

The main goal of this work is to generalize the Bernstein and Pólya theorems to the realm of quantum calculus. Our guiding strategy is to study the convergence of degree elevation of $q$-Bézier curves (i.e., polynomial curves represented in the $q$-Bernstein bases of increasing degrees) on the interval $[0,1]$.

After a brief review of the main quantum tools in Section 2, in Section 3 we show that, unlike the classical case, for $q>1$, the piecewise linear interpolants of the degree elevated $q$-Bézier coefficients of $P$ converge uniformly to a piecewise linear function whose vertices are the values of $P$ on the closure $\overline{\mathscr{A}}_{q}$ of the $q$-lattice $\mathscr{A}_{q}=\left\{q^{-j}, j \in \mathbb{N}\right\}$. A similar result is established for $q<1$, but surprisingly the vertices of the piecewise linear limit are not values of $P$ but rather values of the polynomial whose $q^{-1}$-Bézier coefficients are the $q$-Bézier coefficients of $P$ taken in the reverse order. The $q$-degree elevation algorithm naturally induces the notion of the $q$-Lorentz degree of a given polynomial which is a quantum deformation of its classical Lorentz degree. As an application of the convergence results mentioned above we introduce a notion of $q$ positivity involving the $q$-lattice $\overline{\mathscr{A}_{\max \left(q, q^{-1}\right)}}$ which guarantees that a given polynomial has a finite $q$-Lorentz degree, i.e., its $q$-Bézier coefficients of some degree relative to $(0,1)$ are all non-negative. This result is a $q$-version of the Bernstein theorem.

In Section 4, we study various properties of the quantum Lorentz degrees. We first show that, for $q>1$, the $q$-Lorentz degree of a polynomial is bounded above by the sum of the $q$-Lorentz degrees of its factors. This property fails to be true when $q<1$. We also compare different quantum Lorentz degrees. As a result, the classical Lorentz degree of a polynomial is always greater than (resp., less than) or equal to its $q$-Lorentz degree for any $q>1$ (resp., for any $q<1$ ). Moreover, we generalize a result of Pólya 
and Szegö [27] by showing that, when $q>1$, the $q$-Lorentz degrees of a polynomial with no zeros in a specific disc coincides with its exact degree.

In Section 5, we give upper bounds for the quantum Lorentz degrees, while in Section 6 we generalize the famous Pólya theorem to polynomials positive on the lattice $\mathscr{A}_{q^{-1}}(q>1)$. Embedding the quantum world in the larger mixed quantum-discrete world, corresponding to the $(q, h)$-Bézier curves introduced in [15], enables us to extend the notion of quantum Lorentz degree in Section 7. Nevertheless, since on any admissible interval $(q, h)$-degree elevation coincides with $q$-degree elevation on $[0,1]$, to some extent this extension is not a real generalization. However, this larger framework gives us an opportunity to elaborate a different approach to $q$-degree elevation convergence more in the spirit of geometric design. This approach leads to another extension of the Bernstein theorem which we compare with the one we previously investigated. We conclude in Section 8 with a brief summary of our work along with some comments it inspires, and with an open problem for future research.

\section{Quantum tools}

We provide here a short review of the $q$-calculus. More details can be found in [17].

We begin with some notation. Let $q$ be a positive number. For any real number $a$, the $q$-Pochhammer symbols are defined by setting

$$
(a ; q)_{0}=1,(a ; q)_{n}:=\prod_{k=0}^{n-1}\left(1-a q^{k}\right) .
$$

Using this notation, the $q$-binomial coefficients are defined by setting

$$
\left[\begin{array}{l}
n \\
k
\end{array}\right]_{q}=\frac{(q ; q)_{n}}{(q ; q)_{k}(q ; q)_{n-k}}=\frac{[n]_{q} !}{[k]_{q} ![n-k]_{q} !},
$$

where

$$
[k]_{q} !=[1]_{q}[2]_{q} \ldots[k]_{q}, \quad[0]_{q} !=1,
$$

and $[k]_{q}$ refers to the $q$-integers defined by

$$
[k]_{q}=1+q+\ldots+q^{k-1}=\left\{\begin{array}{ll}
\frac{q^{k}-1}{q-1} & \text { if } q \neq 1 \\
k & \text { if } q=1
\end{array} .\right.
$$

The $q$-binomial coefficients satisfy the recurrence

$$
\left[\begin{array}{l}
n \\
k
\end{array}\right]_{q}=\left[\begin{array}{l}
n-1 \\
k-1
\end{array}\right]_{q}+q^{k}\left[\begin{array}{c}
n-1 \\
k
\end{array}\right]_{q}, \quad 0 \leq k \leq n .
$$

As is usual with ordinary binomial coefficients, it is convenient to introduce the convention that $\left[\begin{array}{l}n \\ k\end{array}\right]_{q}=0$ whenever $k<0$ or $k>n$.

For any real numbers $a$ and $b$, we define $(b-a)_{q}^{n}$ by

$$
(b-a)_{q}^{n}=(b-a)(b-q a) \ldots\left(b-q^{n-1} a\right) .
$$

Given real numbers $a, b$ such that $b \notin\left\{a, q a, \ldots, q^{n-1} a\right\}$, the degree $n q$-Bernstein basis relative to $(a, b)$ is the sequence of polynomials $B_{k}^{n}(.,(a, b) ; q), k=0,1, \ldots, n$, defined by

$$
B_{k}^{n}(x,(a, b) ; q)=\left[\begin{array}{l}
n \\
k
\end{array}\right]_{q} \frac{(x-a)_{q}^{k}(b-x)_{q}^{n-k}}{(b-a)_{q}^{n}}, \quad x \in \mathbb{R} .
$$


This basis is normalized, in the sense that, see [31],

$$
\sum_{k=0}^{n} B_{k}^{n}(\cdot,(a, b) ; q)=\mathbb{I}
$$

where $\mathbb{I}$ denotes the constant function $\mathbb{I}(x)=1$ for all $x$. Concerning the $q$-Bernstein bases relative to $(0,1)$, see also $[23,24,16,22,25]^{1}$.

The notion of $q$-blossoming introduced in [31] is a generalization of the classical notion of blossoming to the $q$-calculus. This generalization is achieved by maintaining the symmetry and the multi-affinity of the classical notion while modifying the diagonal property. More precisely,

Definition 1. Let $\mathbb{P}_{n}$ denote the space of polynomials of degree $n$. Given $P \in \mathbb{P}_{n}$, the unique multi-affine symmetric function $p(. ; q)$ of $n$ variables that satisfies $p(x, q x, \ldots$, $\left.q^{N-1} x ; q\right)=P(x)$ for any $x \in \mathbb{R}$ is called the $q$-blossom of $P$.

When the polynomial $P$ is expressed in the monomial basis as $P(x)=\sum_{k=0}^{n} a_{k} x^{k}$, the $q$-blossom of $P$ is given explicitly by

$$
p\left(u_{1}, u_{2}, \ldots, u_{n} ; q\right)=\sum_{k=0}^{n} a_{k} \frac{\sigma_{k}\left(u_{1}, u_{2}, \ldots, u_{n}\right)}{\sigma_{k}\left(1, q, \ldots, q^{n-1}\right)}, \quad u_{1}, u_{2}, \ldots, u_{n} \in \mathbb{R} .
$$

where $\sigma_{k}$ denotes the $k$ th elementary symmetric function in $n$ variables, that is,

$$
\sigma_{k}\left(u_{1}, \ldots, u_{n}\right)=\sum_{1 \leq i_{1}<i_{2}<\ldots<i_{k} \leq n} u_{i_{1}} u_{i_{2}} \ldots u_{i_{k}} .
$$

Notice that [31]

$$
\sigma_{k}\left(1, q, q^{2}, \ldots, q^{n-1}\right)=q^{k(k-1) / 2}\left[\begin{array}{l}
n \\
k
\end{array}\right]_{q}, \quad 0 \leq k \leq n .
$$

When a polynomial $P$ is expressed in the $q$-Bernstein basis as

$$
P=\sum_{k=0}^{n} p_{k} B_{k}^{n}(.,(a, b) ; q),
$$

the coefficients $p_{k}, k=0, \ldots, n$, called the $q$-Bézier coefficients of $P$ relative to $(a, b)$, are given by [31]

$$
p_{k}=p\left(a q^{k}, a q^{k+1}, \ldots, a q^{n-1}, b, b q, \ldots, b q^{k-1} ; q\right)
$$

where $p(. ; q)$ is the $q$-blossom of the polynomial $P$.

A polynomial $P$ of degree at most $n$ with $q$-Bézier coefficients $p_{0}, p_{1}, \ldots, p_{n}$ relative to $(a, b)$ can also be viewed as a polynomial of degree at most $n+1$ with (provided that we also have $\left.b \neq a q^{n}\right) q$-Bézier coefficients $r_{0}, r_{1} \ldots, r_{n+1}$, relative to $(a, b)$, where $r_{0}=p_{0}, r_{n+1}=p_{n}$ and [14]

$$
r_{i}=\frac{[n+1-i]_{q}}{[n+1]_{q}} p_{i}+q^{n+1-i} \frac{[i]_{q}}{[n+1]_{q}} p_{i-1}, i=1, \ldots, n .
$$

Iterating formula (10) to express the polynomial $P$ in $q$-Bernstein bases of higher degrees is called $q$-degree elevation $[1,15]$. Observe that (10) depends only on $q$, not on $a, b$.

\footnotetext{
${ }^{1}$ Lagrange bases with nodes at $\left\{a q^{j}, j=0,1, \ldots, n\right\}$ are special cases of $q$-Bernstein bases. There is also a relationship between $q$-Bernstein bases relative to $(0,1)$ and Newton bases (See Section 4).
} 
Remark 1. When $q=1$, the $q$-Berntein basis (5) coincides with the classical Bernstein basis (2), the $q$-blossom (7) of a polynomial $P \in \mathbb{P}_{n}$ coincides with its classical blossom giving $P$ by restriction to the diagonal of $\mathbb{R}^{n}$, and the $q$-degree elevation formula (10) is the ordinary degree elevation formula recalled in (3).

\section{Quantum degree elevation and quantum Lorentz degree}

The question addressed in this section is the possible convergence of infinite iteration of the $q$-degree elevation described in (10), when $q$ is a positive number such that $q \neq 1$. This convergence will enable us to derive a $q$-version of Bernstein's theorem.

\section{1. q-Bézier coefficients through degree elevation}

In this subsection we fix a positive number $q \neq 1$. In the lemma below we examine a situation where the $q$-Bézier coefficients have a specific interpretation which will be essential in the study of convergence.

Lemma 1. For any non-zero $a \in \mathbb{R}$, the expansion of any polynomial $P \in \mathbb{P}_{n}$ in the $q$-Bernstein basis relative to $\left(a, a q^{n}\right)$ is given by

$$
P=\sum_{k=0}^{n} P\left(a q^{k}\right) B_{k}^{n}\left(.,\left(a, a q^{n}\right) ; q\right) .
$$

Proof. Let $p(. ; q)$ denote the $q$-blossom of the polynomial $P=\sum_{k=0}^{n} p_{k} B_{k}^{n}\left(.,\left(a, a q^{n}\right) ; q\right)$. Applying (9) with $b=a q^{n}$ yields

$$
p_{k}=p\left(a q^{k}, \ldots, a q^{n-1}, a q^{n}, a q^{n+1}, \ldots, a q^{n+k-1} ; q\right)=P\left(a q^{k}\right) .
$$

Remark 2. Clearly, Lemma 1 means that, when $a \neq 0$, the $q$-Bernstein basis relative to $\left(a, a q^{n}\right)$ coincides with the Lagrange basis associated with the sequence $a q^{j}, j=$ $0, \ldots, n$. This fact was already observed in [25, Section 6, Item 3], where it was derived from the expressions (5) for the $q$-Bernstein bases.

With any sequence $\mathbf{p}:=\left(p_{0}, \ldots, p_{n}\right)$ of real numbers, let us associate the following family of parameterized polynomials by setting:

$$
\mathscr{P}(. ; \mathbf{p} ; \omega)=\sum_{k=0}^{n} p_{k} B_{k}^{n}(.,(\omega, 1) ; q), \quad \omega \notin\left\{q^{-j} \mid j=0, \ldots, n-1\right\} .
$$

Applying degree elevation to this family, we can state:

Proposition 1. For each $\mathbf{p}:=\left(p_{0}, \ldots, p_{n}\right)$, and each $\omega \notin\left\{q^{-j} \mid j=0, \ldots, N-1\right\}$, where $N \geq n$, relative to $(\omega, 1)$ the q-Bézier coefficients of the polynomial $\mathscr{P}(. ; \mathbf{p} ; \omega)$ considered as a polynomial of degree at most $N$, are the values of the polynomial $\mathscr{P}\left(. ; \mathbf{p} ; q^{-N}\right)$ at the points $q^{k-N}, k=0, \ldots, N$, i.e.,

$$
\mathscr{P}(. ; \mathbf{p} ; \omega)=\sum_{k=0}^{N} \mathscr{P}\left(q^{k-N} ; \mathbf{p} ; q^{-N}\right) B_{k}^{N}(.,(\omega, 1) ; q) .
$$



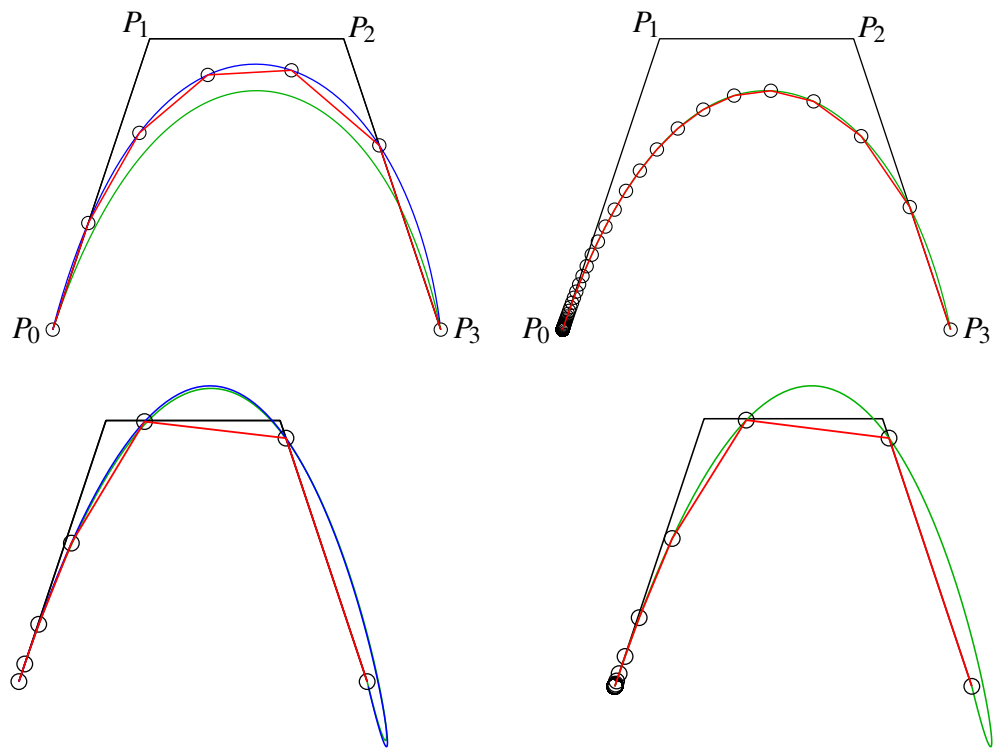

Figure 1: The initial (black) $q$-control polygon of a cubic $q$-Bézier curve on $[0,1]$ (green curve) defined by the sequence $\mathbf{p}=\left(p_{0}, p_{1}, p_{2}, p_{3}\right)$, elevated to degree $N$ (red polygon). The (blue) curve is the cubic $q$-Bézier curve on the interval $\left[q^{-N}, 1\right]$ generated by $\mathscr{P}\left(. ; \mathbf{p} ; q^{-N}\right)$. Left: $N=6$. Right: $N=100$. Top: $q=1.2$. Bottom: $q=2.4$.

Proof. As already observed, the degree elevation formula (10) is independent of the real numbers $a$ and $b$. Accordingly, the $q$-Bézier coefficients relative to $(\omega, 1)$ of the polynomial $\mathscr{P}(. ; \mathbf{p} ; \omega)$ considered as a polynomial of degree at most $N$, are also independent of $\omega$. For $\omega=q^{-N}$, Lemma 1 tells us that the $q$-Bézier-coefficients are equal to

$$
\mathscr{P}\left(q^{k-N} ; \mathbf{p} ; q^{-N}\right), \quad k=0, \ldots, N .
$$

Whence the expansion (12).

\subsection{Convergence: the case $q>1$}

From Proposition 1 we can derive:

Theorem 1. Suppose that $q>1$. Let $P \in \mathbb{P}_{n}$ be expanded in the $q$-Bernstein basis relative to $(0,1)$ of degree $N \geq n$, as

$$
P=\sum_{k=0}^{N} p_{k}^{N} B_{k}^{N}(.,(0,1) ; q), \quad N \geq n .
$$

Then

$$
\lim _{N \rightarrow+\infty} \max _{j=0,1, \ldots, N}\left|P\left(q^{j-N}\right)-p_{j}^{N}\right|=0 .
$$

Proof. Let $p_{0}, \ldots, p_{n}$ denote the initial $q$-Bézier coefficients of $P$ relative to $(0,1)$, i.e., $P=\sum_{k=0}^{N} p_{k} B_{k}^{n}(.,(0,1) ; q)$. Setting $\mathbf{p}=\left(p_{0}, \ldots, p_{n}\right)$, we have $P=\mathscr{P}(. ; \mathbf{p} ; 0)$. According to Proposition 1 , we know that

$$
p_{k}^{N}=\mathscr{P}\left(q^{k-N} ; \mathbf{p} ; q^{-N}\right) \quad \text { for any } N \geq n \text { and for } k=0, \ldots, N .
$$


From the expression (5) for the $q$-Bernstein bases, it is easily seen that, for each $k=0, \ldots, n$, the function $B_{k}^{n}(.,(\omega, 1) ; q)$ converges to $B_{k}^{n}(.,(0,1) ; q)$ when $\omega \rightarrow 0$, uniformly on the interval $[0,1]$. Since

$$
P-\mathscr{P}(. ; \mathbf{p} ; \omega)=\sum_{k=0}^{n} p_{k}\left[B_{k}^{n}(.,(0,1) ; q)-B_{k}^{n}(.,(\omega, 1) ; q)\right]
$$

the function $\mathscr{P}(. ; \mathbf{p} ; \omega)$ converges to $P$ when $\omega \rightarrow 0$, uniformly on $[0,1]$. In particular

$$
\begin{array}{r}
\max _{j=0,1, \ldots, N}\left|P\left(q^{j-N}\right)-p_{j}^{N}\right|=\max _{j=0,1, \ldots, N}\left|P\left(q^{j-N}\right)-\mathscr{P}\left(q^{j-N} ; \mathbf{p} ; q^{-N}\right)\right| \\
\leq\left\|P-\mathscr{P}\left(. ; \mathbf{p} ; q^{-N}\right)\right\|_{\infty}
\end{array}
$$

where $\|\cdot\|_{\infty}$ stands for the uniform norm on the interval $[0,1]$.

Let us interpret Theorem 1 in terms of uniform convergence on $[0,1]$ by introducing parameterisations. For each $N \geq n$, let $\mathbb{L}_{N} ;[0,1] \rightarrow \mathbb{R}$ be defined as follows:

$$
\mathbb{L}_{N}\left(q^{i-N}\right):=p_{i}^{N} \text { for } i=0, \ldots, N,
$$

$\mathbb{L}_{N}$ is constant on $\left[0, q^{-N}\right]$ and affine on $\left[q^{i-N}, q^{i+1-N}\right]$ for $i=0, \ldots, N-1$.

It is easily checked that on $[0,1]$ the sequence $\left(\mathbb{L}_{N}\right)_{N>n}$ converges uniformly to the piecewise linear function $\mathbb{L}$ defined by

$$
\mathbb{L}(0):=P(0), \quad \mathbb{L} \text { is affine on }\left[q^{-j-1}, q^{-j}\right] \text { and } \mathbb{L}\left(q^{-j}\right):=P\left(q^{-j}\right) \text { for all } j \geq 0 .
$$

The previous results are illustrated in Figure 1 , where $\mathbf{p}=\left(p_{0}, p_{1}, p_{2}, p_{3}\right)$ is now a sequence of points in $\mathbb{R}^{2}$, representing the initial (black) $q$-control polygon of the planar parametric cubic curve (green curve) defined by

$$
P(x)=\sum_{k=0}^{3} B_{k}^{3}(x,(0,1) ; q) p_{k}=\sum_{i=0}^{N} B_{i}^{N}(x,(0,1) ; q) p_{i}^{N}, \quad x \in[0,1] .
$$

For short, we refer to this curve as a q-Bézier curve. The (red) polygon with vertices the $p_{i}^{N}, i=0, \ldots, N$, is the final control polygon of the same $q$-Bézier curve, generated by $(N-3)$ degree elevation steps. The left picture corresponds to $N=6$, and the right one to $N=100$. The blue curve is defined by

$$
\mathscr{P}\left(x ; \mathbf{p} ; q^{-N}\right)=\sum_{k=0}^{3} B_{k}^{3}\left(x,\left(q^{-N}, 1\right) ; q\right) p_{k}=\sum_{i=0}^{N} B_{i}^{N}\left(x,\left(q^{-N}, 1\right) ; q\right) p_{i}^{N}, \quad x \in\left[q^{-N}, 1\right]
$$

Observe that, as stated in Proposition 1, the vertices of the red polygon are located on the blue curve. As would be expected from the uniform convergence on $[0,1]$ of $\mathscr{P}\left(. ; \mathbf{p} ; q^{-N}\right)$ to $P$, on the right picture the blue and green curves are no longer distinguishable. The illustrations are obtained with $q=1.2$ (top) and $q=2.4$ (bottom).

\subsection{Convergence: the case $q<1$}

There is a major difference between the cases $q<1$ and $q>1$, namely that

$$
\lim _{N \rightarrow+\infty} q^{-N}=0 \quad \text { if } q>1, \quad \lim _{N \rightarrow+\infty} q^{-N}=+\infty \quad \text { if } q<1 .
$$



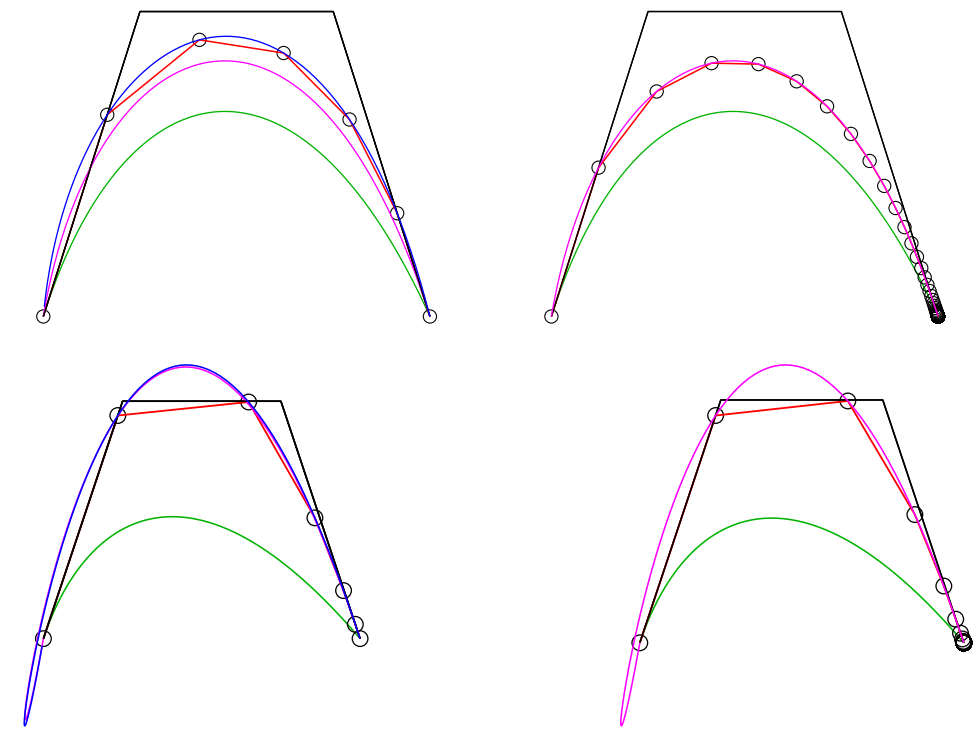

Figure 2: The initial (black) control polygon of a cubic $q$-Bézier curve (green curve) defined by the sequence $\mathbf{p}=\left(p_{0}, p_{1}, p_{2}, p_{3}\right)$, elevated to degree $N$ (red polygon). The pink curve is the cubic $q^{-1}$-Bézier curve with control polygon defined by $\widetilde{\mathbf{p}}:=\left(p_{3}, p_{2}, p_{1}, p_{0}\right)$. The blue curve is the cubic $q^{-1}$-Bézier curve on the interval $\left[q^{N}, 1\right]$ generated by $\mathscr{P}\left(. ; \widetilde{\mathbf{p}} ; q^{N}\right)$. Left: $N=6$. Right: $N=30$. Top: $q=0.8$. Bottom: $q=0.4$.

This difference explains why the arguments developed in the previous subsection are no longer applicable when $q<1$. For any positive $q$ and $a=q^{-n}$, Lemma 1 says that the points

$$
P\left(q^{-n}\right), P\left(q^{-n+1}\right), \ldots, P\left(q^{-1}\right), P(1),
$$

are the $q$-Bézier points of $P$ relative to $\left(q^{-n}, 1\right)$. Lemma 1 also shows that, taken in the reverse order, these points are the $q^{-1}$-Bézier points of $P$ relative to $\left(1, q^{-n}\right)$. Thus when $q<1$, considering the reverse order is quite normal. This observation makes it natural to introduce, for any positive $q$, and any integer $n \geq 0$, the linear map $T_{q}^{n}: \mathbb{P}_{n} \rightarrow$ $\mathbb{P}_{n}$ defined as follows:

$$
\text { if } P=\sum_{k=0}^{n} p_{k} B_{k}^{n}(.,(0,1) ; q) \text { then } T_{q}^{n}(P):=\sum_{k=0}^{n} p_{n-k} B_{k}^{n}\left(.,(0,1) ; \frac{1}{q}\right) \text {. }
$$

Lemma 2. If $\widetilde{P}:=T_{q}^{n}(P)$, then for any $N \geq n$ we have $\widetilde{P}=T_{q}^{N}(P)$.

Proof. It is sufficient to prove that $\widetilde{P}:=T_{q}^{n+1}(P)$. Let us write $P$ as

$$
P=\sum_{k=0}^{n} p_{k} B_{k}^{n}(.,(0,1) ; q)=\sum_{i=0}^{n+1} r_{i} B_{i}^{n+1}(.,(0,1) ; q)
$$

where the sequence $\left(r_{0}, \ldots, \ldots, r_{n+1}\right)$ is derived from the sequence $\left(p_{0}, \ldots, p_{n}\right)$ through the $q$-degree elevation formulas (10). Taking account of the obvious relation

$$
[i]_{\frac{1}{q}}=[i]_{q} \frac{1}{q^{i-1}}, \quad i \geq 1,
$$


it is easily checked that the sequence $\left(r_{n+1}, r_{n}, \ldots, r_{0}\right)$ is derived from the sequence $\left(p_{n}, p_{n-1}, \ldots, p_{0}\right)$ through the degree elevation formulas generated by replacing $q$ by $\frac{1}{q}$ in (10).

For any polynomial $P$, the previous lemma gives sense to the notation $T_{q}(P)$ defined as $T_{q}^{N}(P)$ for any $N$ greater than or equal to the exact degree of $P$. Note that if $q=1$, then $T_{1}(P)(x)=P(1-x)$ for all $x$.

Theorem 2. Let $q<1$ and let $P \in \mathbb{P}_{n}$ be expanded in the $q$-Bernstein basis relative to $(0,1)$ of degree $N \geq n$, as

$$
P=\sum_{k=0}^{N} p_{k}^{N} B_{k}^{N}(.,(0,1) ; q), \quad N \geq n .
$$

Then

$$
\lim _{N \rightarrow+\infty} \max _{j=0,1, \ldots, N}\left|T_{q}(P)\left(q^{j}\right)-p_{j}^{N}\right|=0 .
$$

Proof. We can apply Theorem 1 to the pair $\left(\widetilde{P}, q^{-1}\right)$, with $\widetilde{P}:=T_{q}(P)=T_{q}^{n}(P)$. From Lemma 2, we know that

$$
\widetilde{P}=\sum_{k=0}^{N} p_{N-k}^{N} B_{k}^{N}\left(.,(0,1) ; q^{-1}\right), \quad N \geq n .
$$

From (13) we can thus deduce that

$$
\lim _{N \rightarrow+\infty} \max _{j=0,1, \ldots, N}\left|\widetilde{P}\left(\left(q^{-1}\right)^{j-N}\right)-p_{N-j}^{N}\right|=0,
$$

which is the same as (15).

As previously, we can interpret Theorem 2 in terms of uniform convergence on $[0,1]$. For each $N \geq n$, let $\widetilde{\mathbb{L}}_{N} ;[0,1] \rightarrow \mathbb{R}$ be defined as follows:

$$
\begin{gathered}
\widetilde{\mathbb{L}}_{N}\left(q^{i}\right):=p_{i}^{N} \text { for } i=0, \ldots, N, \\
\widetilde{\mathbb{L}}_{N} \text { is constant on }\left[0, q^{N}\right] \text { and affine on }\left[q^{i+1}, q^{i}\right] \text { for } i=N-1, \ldots, 0 .
\end{gathered}
$$

Then the sequence $\left(\mathbb{L}_{N}\right)_{N \geq n}$ converges uniformly on $[0,1]$ to the piecewise linear function $\widetilde{\mathbb{L}}$ defined by

$$
\widetilde{\mathbb{L}}(0):=P(1), \quad \widetilde{\mathbb{L}} \text { is affine on }\left[q^{j+1}, q^{j}\right] \text { and } \widetilde{\mathbb{L}}\left(q^{j}\right):=T_{q}(P)\left(q^{j}\right) \text { for all } j \geq 0 \text {. }
$$

This convergence is illustrated in Figure 2 using planar curves, with successively $q=$ 0.8 (top) and $q=0.4$ (bottom). In this case we show the $q$-Bézier curve generated by the cubic polynomial function with initial $q$-control polygon defined by $\mathbf{p}=\left(p_{0}, p_{1}, p_{2}, p_{3}\right)$ (green curve), along with the $q^{-1}$-Bézier curve with initial $q^{-1}$-control polygon defined by $\widetilde{\mathbf{p}}=\left(p_{3}, p_{2}, p_{1}, p_{0}\right)$ (pink curve). The vertices of the final $q$-control polygon are located on the blue curve (visible only for $N=6$ ) defined on $\left[q^{N}, 1\right]$ by the function $\mathscr{P}\left(. ; \widetilde{\mathbf{p}} ; q^{N}\right)$. 


\section{4. q-Lorentz degree and q-positivity}

Throughout this subsection we consider a fixed polynomial $P$ of exact degree $n$, expanded as

$$
P=\sum_{k=0}^{N} p_{k}^{N} B_{k}^{N}(.,(0,1) ; q), \quad N \geq n .
$$

Definition 2. For any positive $q$, the $q$-Lorentz degree of $P$, denoted as $L_{q}(P)$, is defined by

$$
L_{q}(P):=\inf \left\{N \geq n \mid p_{k}^{N} \geq 0 \text { for } k=0, \ldots, N\right\} .
$$

From the previous definition, $L_{q}(P)=+\infty$ means that, for any $N \geq n$, there exists at least one integer $k \in\{0,1, \ldots, N\}$ such that $p_{k}^{N}<0$. From Lemma 2 we can state that:

$$
L_{q}(P)=L_{q^{-1}}\left(T_{q}(P)\right) \quad \text { for each positive } q .
$$

The 1-Lorentz degree $L_{1}(P)$ is the classical Lorentz degree of $P$, also denoted by $L_{[0,1]}(P)$. From Bernstein's theorem we know that the positivity of $P$ on $[0,1]$ is sufficient to ensure that $L_{[0,1]}(P)<+\infty$. Let us now consider the case $q \neq 1$. To establish a similar sufficient condition, we denote by $\mathscr{A}_{q}$ and $\mathscr{B}_{q}$ the $q$-lattices defined by

$$
\mathscr{A}_{q}:=\left\{q^{-j}, j \in \mathbb{N}\right\}, \quad \mathscr{B}_{q}:=\left\{q^{j}, j \in \mathbb{N}\right\}=\mathscr{A}_{q^{-1}} .
$$

We also consider the closures $\overline{\mathscr{A}_{q}}=\mathscr{A}_{q} \cup\{0\} \subset[0,1]$ (when $q>1$ ), and $\overline{\mathscr{B}_{q}}=\mathscr{B}_{q} \cup$ $\{0\} \subset[0,1]$ (when $q<1)$.

Definition 3. Given a positive number $q \neq 1$, the polynomial $P$ is said to be q-positive

- when $P$ is positive on the $q$-lattice $\overline{\mathscr{A}_{q}}$ if $q>1$;

- when the polynomial $T_{q}(P)$ is positive on the $q$-lattice $\overline{\mathscr{B}} q$ if $q<1$, i.e., when $T_{q}(P)$ is $q^{-1}$-positive.

With this definition, the following direct consequence of Theorems 1 and 2 can be seen as a generalization of Bernstein's theorem.

Theorem 3. Given a positive number $q \neq 1$, the following three properties are equivalent:

(i) the polynomial $P$ is q-positive;

(ii) $P(0)>0, P(1)>0$, and the $q$-Lorentz degree of $P$ is finite;

(iii) for $N$ sufficiently large, all q-Bézier coefficients $p_{k}^{N}, k=0, \ldots, N$, are positive.

Proof. It is sufficient to prove this theorem for $q>1$. Assume that (i) holds. Then the minimum of $P$ on the compact set $\overline{\mathscr{A}}_{q}$ is positive. From (13) we can conclude that (iii) is satisfied. Conversely, if (iii) is satisfied, select an integer $N^{*} \geq n$ such that all $q$-Bézier coefficients $p_{k}^{N^{*}}, k=0, \ldots, N^{*}$, are positive, and let $p^{*}$ denote the positive number

$$
p^{*}:=\min \left\{p_{k}^{N^{*}} \mid k=0, \ldots, N^{*}\right\} .
$$

Applying the $q$-degree elevation formulas (10), we can deduce that

$$
\min \left\{p_{k}^{N} \mid k=0, \ldots, N\right\} \geq p^{*}>0 \quad \text { for each } N \geq N^{*} .
$$

Accordingly, thanks to (13) one can find an integer $\widetilde{N}^{*} \geq N^{*}$ such that

$$
\min \left\{P\left(q^{-j}\right) \mid j=0, \ldots, N\right\}>0 \quad \text { for each } N \geq \widetilde{N}^{*} .
$$


Along with the fact that $P(0)>0$, this means that $P$ is $q$-positive.

The implication (iii) $\Rightarrow$ (ii) being trivial, it only remains to show that (ii) $\Rightarrow$ (iii). Suppose that (ii) is satisfied and let $N$ denote the integer $L_{q}(P)$. From (ii) we know that the extreme $q$-Bézier coefficients $p_{0}^{N}, p_{N}^{N}$ are positive and that $p_{1}^{N}, \ldots, p_{N-1}^{N}$ are nonnegative. Suppose that at least one of them is zero. Then we can consider two integers $i \geq 0, r \geq 1$, such that

$$
p_{i}^{N}>0, \quad p_{i+1}^{N}=p_{i+2}^{N}=\cdots=p_{i+r}^{N}=0, \quad p_{i+r+1}^{N}>0 .
$$

The $q$-degree elevation formulas (10) imply that

$$
p_{i}^{N+1}, p_{i+1}^{N+1}>0, \quad p_{i+2}^{N+1}=\cdots=p_{i+r}^{N+1}=0, \quad p_{i+r+1}^{N+1}, p_{i+r+2}^{N+1}>0
$$

Therefore the maximum number of consecutive $q$-Bézier coefficients of $P$ that are zero diminishes whenever we apply one step of $q$-degree elevation. Accordingly, we can find $\bar{N} \geq N$ such that all $q$-Bézier coefficients $p_{k}^{\bar{N}}, k=0, \ldots, \bar{N}$, are positive. Hence (iii) is satisfied.

Remark 3. Observe that, when $q \neq 1$, the distance between any two consecutive points in the lattice involved in the $q$-positivity is bounded above by $|q-1|$. Therefore, this distance goes to 0 when $q$ gets closer and closer to 1 . Accordingly, the 1-positivity of a polynomial $P$, in the sense of the limit of the $q$-positivity when $q \neq 1$ tends to 1 , should be understood as the positivity of $P$ on $[0,1]$. From this point of view, Theorem 3 definitely provides us with a generalisation of Bernstein's theorem on $[0,1]$, namely: for any positive $q$, if the polynomial $P$ is q-positive, then $P$ admits a representation of the form

$$
P(x)=\sum_{k=0}^{N} a_{k} x^{k}(1-x)(1-q x) \ldots\left(1-q^{n-k-1} x\right), \quad \text { with } a_{k} \geq 0 \text { for } k=0, \ldots, N .
$$

\section{Properties of quantum Lorentz degrees}

In the present section we establish some properties concerning the $q$-Lorentz degrees. These properties have in common to be somehow attached to a nice interpretation of $q$-Bézier coefficients established in the first subsection.

\section{1. q-Bézier coefficients as divided differences}

Throughout the present subsection, $q$ is a fixed positive number. All results developed in the present section rely on an elementary tool that we describe now.

A non-negative integer $N$ being given, denote by $\Theta_{N}: \mathbb{P}_{N} \rightarrow \mathbb{P}_{N}$ the linear involution defined by

$$
\Theta_{N}(P)(y):=y^{N} P(1 / y) \text { for all } y \neq 0, \quad P \in \mathbb{P}_{N} .
$$

Notice that in the monomial basis $P$ and $\Theta_{N}(P)$ have the same coefficients, but taken in reverse orders. Consider the basis $\left(Q_{0}, \ldots, Q_{N}\right)$ of $\mathbb{P}_{N}$ defined by

$$
Q_{k}(x):=x^{N-k}(1-x)_{q}^{k}, \quad x \in \mathbb{R}, \quad k=0, \ldots, N,
$$

and denote by $\left(P_{0}, \ldots, P_{N}\right)$ its image under $\Theta_{N}$. Clearly, for all $y \in \mathbb{R}$,

$$
P_{k}(y):=\Theta_{N}\left(Q_{k}\right)(y)=(y-1)_{q}^{k}=(y-1)(y-q) \ldots\left(y-q^{k-1}\right), \quad k=0, \ldots, N .
$$


In other words, under the linear map $\Theta_{N}$, the basis $\left(Q_{0}, \ldots, Q_{N}\right)$ is transformed into the Newton basis attached to the sequence $\left(1, q, \ldots, q^{N-1}\right)$. As is well known, expansions in this Newton basis are obtained via divided differences [7, 13]

$$
P=\sum_{k=0}^{N}\left(\left[1, q, \ldots, q^{k}\right] P\right) P_{k} \quad \text { for all } P \in \mathbb{P}_{N},
$$

where, for any real numbers $x_{1}, \ldots, x_{n}$ and any function $F$ defined on an interval containing these real numbers, $\left[x_{1}, \ldots, x_{n}\right] F$ refers to the divided difference of $F$ based on $x_{1}, \ldots, x_{n}$. Accordingly, the link between the $q$-Bernstein basis relative to $(0,1)$ and the Newton basis $\left(P_{0}, \ldots, P_{N}\right)$ readily follows from (18) and from the equality

$$
B_{N-k}^{N}(\bullet,(0,1) ; q)=\left[\begin{array}{c}
N \\
k
\end{array}\right]_{q} Q_{k}, \quad k=0, \ldots, N,
$$

which leads to the crucial property stated in the following proposition:

Proposition 2. Let a polynomial $P \in \mathbb{P}_{N}$ be expressed in the $q$-Bernstein basis relative to $(0,1)$ as

$$
P=\sum_{k=0}^{N} p_{k} B_{k}^{N}(.,(0,1) ; q) .
$$

The q-Bézier coefficients of $P$ relative to $(0,1)$ are given by

$$
p_{N-k}\left[\begin{array}{c}
N \\
k
\end{array}\right]_{q}=\left[1, q, \ldots, q^{k}\right] \Theta_{N}(P), \quad k=0,1, \ldots, N,
$$

The previous proposition has been established strictly in view of developing the properties of $q$-Lorentz degrees. Nonetheless, we would like to indicate some observations that can be readily derived from Proposition 2.

Corollary 1. The q-binomial coefficients $\left[\begin{array}{l}N \\ k\end{array}\right]_{q}$ can be expressed as follows:

$$
\left[\begin{array}{c}
N \\
k
\end{array}\right]_{q}=\left[1, q, \ldots, q^{k}\right] R_{N}=\left[1, q, \ldots, q^{N-k}\right] R_{N}, \quad k=0, \ldots, N,
$$

where $R_{N}$ denotes the monomial $R_{N}(y)=y^{N}$.

Proof. Observe that $R_{N}:=\Theta_{N}(\mathbb{I})$. Accordingly, (21) is obtained by applying (20) with $P=\mathbb{I}$.

Remark 4. Formula (21) extends the classical equality $\left(\begin{array}{c}N \\ k\end{array}\right)=\frac{P^{(k)}(1)}{k !}$ obtained with $q=1$. With the help of (21) we can now rewrite the relations (20) as ratios of divided differences:

$$
p_{N-k}=\frac{\left[1, q, \ldots, q^{k}\right] \Theta_{N}(P)}{\left[1, q, \ldots, q^{k}\right] R_{N}}, \quad k=0,1, \ldots, N .
$$

Furthermore, with the interpretation (21), observe that the recurrence relation (4) can be viewed as the effect of the Leibniz formula for divided differences:

$$
\begin{aligned}
{\left[1, q, \ldots, q^{k}\right] R_{N+1} } & =\left[1, q, \ldots, q^{k}\right]\left(R_{N} R_{1}\right)=\sum_{i=0}^{k}\left(\left[1, q, \ldots, q^{i}\right] R_{N}\right)\left(\left[q^{i}, \ldots, q^{k}\right] R_{1}\right) \\
& =\left[1, q, \ldots, q^{k-1}\right] R_{N}+\left(\left[1, q, \ldots, q^{k}\right] R_{N}\right) R_{1}\left(q^{k}\right) .
\end{aligned}
$$


The sequence of linear maps $\Theta_{n}, n \geq 0$, preserves the algebra structure of polynomials in the obvious sense that, for any non-negative integers $M, N$,

$$
\Theta_{M+N}(P Q)=\Theta_{M}(P) \Theta_{N}(Q) \text { for all } P \in \mathbb{P}_{M}, Q \in \mathbb{P}_{N}
$$

As an obvious special case of (23), considering $P \in \mathbb{P}_{n}$ as an element of $\mathbb{P}_{n+r}$ for some $r \geq 1$, we obtain with the notation introduced in Corollary 1 :

$$
\Theta_{n+r}(P)=\Theta_{n}(P) \Theta_{r}(\mathbb{I})=\Theta_{n}(P) R_{r}, \quad \text { for any } P \in \mathbb{P}_{n} \text {, and any } r \geq 0 .
$$

Accordingly, (20) provides us with explicit expressions for the final $q$-Bézier coefficients of a polynomial after any number of $q$-degree elevation steps in terms of its initial $q$-Bézier coefficients.

Corollary 2. Let $P \in \mathbb{P}_{n}$ be expanded as

$$
P=\sum_{k=0}^{n} p_{k} B_{k}^{n}(.,(0,1) ; q)=\sum_{k=0}^{N} p_{k}^{N} B_{k}^{N}(.,(0,1) ; q), \quad \text { with } N \geq n .
$$

Then, the final $q$-Bézier coefficients $p_{0}^{N}, \ldots, p_{N}^{N}$ are obtained as convex combinations of the initial $q$-Bézier coefficients $p_{0}, \ldots, p_{n}$, as follows:

$$
p_{N-i}^{N}=\sum_{k=0}^{i} \frac{q^{k(N-n-i+k)}\left[\begin{array}{c}
n \\
k
\end{array}\right]_{q}\left[\begin{array}{c}
N-n \\
i-k
\end{array}\right]_{q}}{\left[\begin{array}{c}
N \\
i
\end{array}\right]_{q}} p_{n-k}, \quad i=0, \ldots, N .
$$

Proof. Set $Q:=\Theta_{n}(P)$. Using (24) and (20), along with the Leibniz formula, we can successively write

$$
\begin{aligned}
p_{N-i}^{N}\left[\begin{array}{c}
N \\
i
\end{array}\right]_{q} & =\left[1, q, \ldots, q^{i}\right]\left(Q R_{N-n}\right)=\sum_{k=0}^{i}\left(\left[1, q, \ldots, q^{k}\right] Q\right)\left(\left[q^{k}, q^{k+1}, \ldots, q^{i}\right] R_{N-n}\right) \\
& =\sum_{k=0}^{i}\left(p_{n-k}\left[\begin{array}{l}
n \\
k
\end{array}\right]_{q}\right)\left(\left[q^{k}, q^{k+1}, \ldots, q^{i}\right] R_{N-n}\right) .
\end{aligned}
$$

Taking account of the following equality, valid for all non-negative integers $\ell, s, m$,

$$
\left[q^{\ell}, q^{\ell+1}, \ldots, q^{\ell+s}\right] R_{m}=q^{\ell(m-s)}\left[\begin{array}{c}
m \\
s
\end{array}\right]_{q}
$$

this yields formula (25).

When $N=n+1$, relation (25) coincides with (10).

\section{2. q-Lorentz degree of a product}

We now have at our disposal efficient tools to develop the properties of quantum Lorentz degrees. In particular, the essential observation (23) will help answer the natural question: how does the $q$-Lorentz degree behave with respect to the product of polynomials? With this problem in view, we start with the following lemma.

Lemma 3. For any $q>1$ and for any non-negative integers $M, N, k, \ell$, such that $0 \leq k \leq$ $M, 0 \leq \ell \leq N$, all the $q$-Bézier coefficients of the product $B_{k}^{M}(.,(0,1) ; q) B_{\ell}^{N}(.,(0,1) ; q)$, in the degree $(M+N) q$-Bernstein basis relative to $(0,1)$ are non-negative. 
Proof. Let $k, \ell$ be any two integers such that $0 \leq k \leq M, 0 \leq \ell \leq N$. Without loss of generality we can assume that $k \geq \ell$.

Via (23), (18), and (19) we obtain

$$
\Theta_{M+N}\left(B_{M-k}^{M}(.,(0,1) ; q) B_{N-\ell}^{N}(.,(0,1) ; q)\right)=\left[\begin{array}{c}
M \\
k
\end{array}\right]_{q}\left[\begin{array}{c}
N \\
\ell
\end{array}\right]_{q} P_{k} P_{\ell} .
$$

Accordingly, on account of (20), proving the claim consists in proving that all the divided differences

$$
\left[1, q, \ldots, q^{i}\right]\left(P_{k} P_{\ell}\right), \quad i=0, \ldots, N+M,
$$

are non-negative. Now the Leibniz formula for divided differences yields:

$$
\left[1, q, \ldots, q^{i}\right]\left(P_{k} P_{\ell}\right)=\sum_{j=0}^{i}\left(\left[1, q, \ldots, q^{j}\right] P_{k}\right)\left(\left[q^{j}, \ldots, q^{i}\right] P_{\ell}\right) .
$$

Since $1, q, \ldots, q^{k-1}$ are the zeros of the degree $k$ polynomial $P_{k}$, we have

$$
\left[1, q, \ldots, q^{j}\right] P_{k}=\delta_{k, j}, \quad j \geq 0 .
$$

Therefore, (26) reduces to

$$
\left[1, q, \ldots, q^{i}\right]\left(P_{k} P_{\ell}\right)= \begin{cases}{\left[q^{k}, \ldots, q^{i}\right] P_{\ell}} & \text { for } k \leq i \leq k+\ell \\ 0 & \text { otherwise }\end{cases}
$$

Moreover, for any $i$ such that $k \leq i \leq k+\ell$, there exists a real number $\eta_{k, i} \in\left[q^{k}, q^{i}\right]$ such that

$$
\left[q^{k}, q^{k+1}, \ldots, q^{i}\right] P_{\ell}=\frac{P_{\ell}^{(i-k)}\left(\eta_{k, i}\right)}{(i-k) !} .
$$

The expression for $P_{\ell}$ makes it clear that, for $j \leq \ell$, all its derivatives $P_{\ell}^{(j)}, j \leq \ell$, are positive on the interval $] q^{\ell-1},+\infty[$. Our assumption that $k \geq \ell$ thus ensures that all the quantities in (28), $k \leq i \leq k+\ell$, are positive.

Lemma 3 enables us to prove the following result.

Theorem 4. Given any $q>1$ and any polynomials $Q, R$, we have

$$
L_{q}(Q R) \leq L_{q}(Q)+L_{q}(R) .
$$

Proof. The claimed relation (29) is trivially satisfied when its right-hand side is infinite. We thus suppose that $L_{q}(Q)=M$ and $L_{q}(R)=N$. Then

$$
Q=\sum_{k=0}^{M} q_{k} B_{k}^{M}(.,(0,1) ; q), \quad R=\sum_{\ell=0}^{N} r_{\ell} B_{\ell}^{N}(.,(0,1) ; q)
$$

where $q_{k}, k=1, \ldots, M$, and $r_{\ell}, \ell=1, \ldots, N$, are non-negative real numbers. Hence,

$$
Q R=\sum_{\substack{0 \leq k \leq M \\ 0 \leq \ell \leq N}} q_{k} r_{\ell} B_{k}^{M}(.,(0,1) ; q) B_{\ell}^{N}(.,(0,1) ; q) .
$$

Lemma 3 ensures that the expansion of $Q R$ in the degree $(M+N) q$-Bernstein basis relative to $(0,1)$ has only non-negative coefficients. This concludes the proof. 
Remark 5. We would like to draw the reader's attention to the fact that formula (29) is not valid for $q<1$. This is due to Lemma 3 itself not being valid for $q<1$. Indeed, in the proof of Lemma 3, for $q<1$ everything remains valid up to formula (28), except that we now have to write $\eta_{k, i} \in\left[q^{i}, q^{k}\right]$. Take $i=k+\ell-1$. Then we are dealing with the derivative of order $(\ell-1)$ of the polynomial $P_{\ell}$. Supposing that $\ell \geq 2$, this derivative is a linear combination with positive integer coefficients of the degree one polynomials $y-q^{j}, j=0, \ldots, \ell-1$. The assumption $k \geq \ell$ used in the proof of Lemma 3 implies that $P_{\ell}^{(i-k)}\left(\eta_{k, i}\right)=P_{\ell}^{(\ell-1)}\left(\eta_{k, i}\right)$ is negative. Accordingly, the $q$-Bézier coefficient of index $i$ of the product $B_{M-k}^{M}(.,(0,1) ; q) B_{N-\ell}^{N}(.,(0,1) ; q)$ considered as a polynomial of degree $(M+N)$ is negative. In other words, when $q<1$ we have

$$
\begin{aligned}
& L_{q}\left(B_{M-k}^{M}(.,(0,1) ; q) B_{N-\ell}^{N}(.,(0,1) ; q)\right)>M+N \\
& =L_{q}\left(B_{M-k}^{M}(.,(0,1) ; q)\right)+L_{Q}\left(B_{N-\ell}^{N}(.,(0,1) ; q)\right),
\end{aligned}
$$

which contradicts (29).

\subsection{Comparison of quantum Lorentz degrees}

A polynomial $P$ is allocated infinitely many different quantum Lorentz degrees, namely, all (possibly infinite) quantities $L_{q}(P), q>0, q \neq 1$. For the same polynomial, can we compare different quantum Lorentz degrees? The following result will answer this question.

Proposition 3. Fix $q \geq 1$. Then, for any positive $\bar{q}$ and for any non-negative integer $N$, the following properties are equivalent:

(i) for each $k=0,1, \ldots, N$, all $\bar{q}$-Bézier coefficients of $B_{k}^{N}(.,(0,1) ; q)$ are positive;

(ii) $\bar{q}>q^{N-1}$.

Proof. On account of the obvious equalities

$$
B_{N}^{N}(.,(0,1) ; q)=B_{N}^{N}(.,(0,1) ; \bar{q}) \quad \text { and } \quad B_{N-1}^{N}(.,(0,1) ; q)=\frac{[N]_{q}}{[N]_{\bar{q}}} B_{N-1}^{N}(.,(0,1) ; \bar{q})
$$

in (i) it is sufficient to consider only the basis functions $B_{N-k}^{N}(.,(0,1) ; q), k=2, \ldots, N$. Applying (18) and (19), Proposition 2 states that

$$
B_{N-k}^{N}(.,(0,1) ; q)=\sum_{i=1}^{k} \frac{\left[\begin{array}{c}
N \\
k
\end{array}\right]_{q}}{\left[\begin{array}{c}
N \\
i
\end{array}\right]_{\bar{q}}}\left(\left[1, \bar{q}, \ldots, \bar{q}^{i}\right] P_{k}\right) B_{N-i}^{N}(.,(0,1) ; \bar{q}), \quad k=2, \ldots, N,
$$

where the polynomial $P_{k}$ is defined in (18). The Leibniz rule shows that

$$
\left[1, \bar{q}, \ldots, \bar{q}^{i}\right] P_{k}=\left[\bar{q}, \bar{q}^{2}, \ldots, \bar{q}^{i}\right] P_{k}^{*}, \quad \text { for } k=2, \ldots, N \text { and } i=1, \ldots, k
$$

where $P_{k}^{*}(y)=(y-q)\left(y-q^{2}\right) \ldots\left(y-q^{k-1}\right)$. Suppose that condition (i) holds. This implies in particular that

$$
[\bar{q}] P_{k}^{*}=P_{k}^{*}(\bar{q})>0, \quad \text { for } \quad k=2, \ldots, N,
$$

that is,

$$
\bar{q}-q>0, \quad(\bar{q}-q)\left(\bar{q}-q^{2}\right)>0, \quad \ldots, \quad(\bar{q}-q)\left(\bar{q}-q^{2}\right) \ldots\left(\bar{q}-\bar{q}^{N-1}\right)>0 .
$$


Condition (ii) is therefore satisfied. Conversely, suppose that (ii) holds. For $2 \leq k \leq N$ and $1 \leq i \leq k$, there exists $\xi_{k, i-1} \in\left[\bar{q}, \bar{q}^{i}\right]$ such that

$$
\left[\bar{q}, \bar{q}^{2}, \ldots, \bar{q}^{i}\right] P_{k}^{*}=\frac{P_{k}^{*(i-1)}\left(\xi_{k, i-1}\right)}{(i-1) !} .
$$

Since $\bar{q}>q^{N-1}$, all the derivatives involved in (30) are positive on the interval $[\bar{q},+\infty[$. This proves (i).

The first immediate consequence of Proposition 3 is stated next.

Corollary 3. Let $P:=\sum_{k=0}^{n} p_{k} B_{k}^{n}(.,(0,1) ; q)$ have non-negative q-Bézier coefficients $p_{0}, \ldots, p_{n}$ for some $q \geq 1$. Then, for any $\bar{q} \geq q^{n-1}$, the $\bar{q}$-Bézier coefficients of $P$ relative to $(0,1)$ are non-negative.

Proof. Proposition 3 implies that, for $\bar{q} \geq q^{n-1}$ and for $k=0, \ldots, n$, the $q$-Bernstein basis function $B_{k}^{n}(.,(0,1) ; q)$ is expanded in the $\bar{q}$-Bernstein basis $B_{j}^{n}(.,(0,1) ; \bar{q}), j=$ $0, \ldots, n$, with non-negative coefficients. Since $p_{0}, \ldots, p_{n}$ are non-negative, the equality $P:=\sum_{k=0}^{n} p_{k} B_{k}^{n}(.,(0,1) ; q)$ shows that $P$ in turn is expanded with non-negative coefficients in the basis $B_{j}^{n}(.,(0,1) ; \bar{q}), j=0, \ldots, n$.

We can apply the previous corollary to compare the $q$-Lorentz degree with another quantum Lorentz degree.

Corollary 4. Any polynomial P satisfies

$$
L_{\bar{q}}(P) \leq L_{q}(P) \quad \text { for any } \bar{q} \geq q^{L_{q}(P)-1} .
$$

Proof. Apply Corollary 3 with $n:=L_{q}(P)$ when $L_{q}(P)<+\infty$.

As a special case of (31) we obtain the following striking formula:

$$
L_{q} L_{q(P)-1} \leq L_{q}(P) \quad \text { for every polynomial } P .
$$

Finally, Proposition 3 leads to the following comparison between the classical Lorentz degree and its quantum counterparts.

Theorem 5. Let $q, \bar{q}$ be two real numbers such that $0<\bar{q}<1<q$. Then for any polynomial $P$

$$
L_{q}(P) \leq L_{[0,1]}(P) \leq L_{\bar{q}}(P) .
$$

Proof. The left inequality is obtained by first exchanging the roles of $q$ and $\bar{q}$ and then taking $\bar{q}=1$ in relation (31).

Let us now prove the right inequality. Here we shall make use of total positivity. First recall that a basis $\left(V_{0}, \ldots, V_{N}\right)$ of $\mathbb{P}_{N}$ is said to be totally positive on $[0,1]$ if, for any sequence of points $0 \leq x_{0}<x_{1}<\cdots<x_{N} \leq 1$, the collocation matrix $\left(V_{j}\left(x_{i}\right)\right)_{0 \leq i, j \leq N}$ is totally positive, i.e., all its minors are non-negative. Among all bases of $\mathbb{P}_{N}$ which are both normalized and totally positive on $[0,1]$, the classical Bernstein basis is the optimal one, in the sense that, for any other basis $\left(V_{0}, \ldots, V_{N}\right)$ of $\mathbb{P}_{N}$ which is normalized and totally positive on $[0,1]$, there exists a corner-cutting algorithm transforming the coordinates of any $P \in \mathbb{P}_{N}$ in the basis $\left(V_{0}, \ldots, V_{N}\right)$ into the Bézier coefficients of $P$ relative to $(0,1)$. For more details on this subject see [19] and also [6]. Since $\bar{q}<1$, the $\bar{q}$-Bernstein basis relative to $(0,1)$ too is normalized and totally positive on $[0,1]$ 
[16]. Given a polynomial $P$ such that $L_{\bar{q}}(P)<+\infty$, take $N=L_{\bar{q}}(P)$. Consider the two expansions

$$
P=\sum_{k=0}^{N} \bar{p}_{k}^{N} B_{k}^{N}(.,(0,1) ; \bar{q})=\sum_{k=0}^{N} p_{k}^{N} B_{k}^{N}(.,(0,1) ; 1) .
$$

All the $\bar{q}$-Bézier coefficients $\bar{p}_{k}^{N}, k=0, \ldots, N$, are non-negative. Since the Bézier coefficients $p_{k}^{N}, k=0, \ldots, N$, are generated from the $\bar{q}$-Bézier coefficients $\bar{p}_{k}^{N}$ by corner cutting, they must themselves be non-negative. Accordingly, $N \geq L_{[0,1]}(P)$, which proves the right-hand side of the inequality (33).

\subsection{Degree and q-Lorentz degree}

In [27], Pólya and Szegö show that: if a polynomial has no zeros in the closed disc centered at $z=1 / 2$ and with radius $1 / 2$, its classical Lorentz degree coincides with its degree. The left inequality in (33) proves that this property remains valid for the $q$-Lorentz degree when $q>1$. As a matter of fact, Theorem 6 below shows that we can even reduce the size of the disc.

Theorem 6. Let a polynomial $P$ satisfy $P(0)>0, P(1)>0$. Suppose that there exists a real number $q>1$ such that $P$ has no zero in a closed disc $\mathbb{D}_{q}$ with center $1 /(1+q)$ and radius $1 /(1+q)$, i.e.,

$$
\mathbb{D}_{q}=\left\{z \in \mathbb{C}|| z-\frac{1}{1+q} \mid \leq \frac{1}{1+q}\right\} .
$$

Then the q-Lorentz degree of $P$ coincides with the exact degree of $P$.

Proof. Let $n$ be the exact degree of $P$, and let $p_{0}, \ldots, p_{n}$ be the $q$-Bézier coefficients of $P$ relative to $(0,1)$. Since $P(0)>0$ and $P(1)>0$, we need to consider only the nonextreme coefficients $p_{1}, \ldots, p_{n-1}$. The result is thus trivially true for $n \leq 1$. Moreover, since $P(0)>0$ and $P(1)>0$, the number of zeros of $P$ in the open interval ]0,1 [, counting multiplicities, is even. Since, in addition, $P$ has no zero in the disc $\mathbb{D}_{q}$, all these zeros are located in the open interval $] 2 /(1+q), 1[$. Accordingly, we can decompose $P$ as

$$
P=P_{1} P_{2} \ldots P_{m},
$$

where, for each $k=1,2, \ldots, m, P_{k}$ is

- either a polynomial of exact degree 1 with no zeros in the interval $[0,1]$;

- or a polynomial of exact degree 2 with no real zeros;

- or a polynomial of exact degree 2 with two zeros in the interval $] 2 /(1+q), 1[$.

Moreover, without loss of generality, we can assume that each polynomial $P_{k}$ satisfies $P_{k}(0)>0$ and $P_{k}(1)>0$. In the first case, we automatically have $L_{q}\left(P_{k}\right)=1$.

It is actually sufficient to prove the claim when $n=2$. Indeed, if this is proved, then, according to Theorem 4, we will have

$$
L_{q}(P) \leq \sum_{k=1}^{m} L_{q}\left(P_{k}\right)=n,
$$

since, for each $k=1, \ldots, m, L_{q}\left(P_{k}\right)$ will coincide with the exact degree of $P_{k}$.

Let us thus assume that $n=2$. First consider the case where $P$ has non-real zeros, i.e.,

$$
P(x)=A\left(x-z_{1}\right)\left(x-\bar{z}_{1}\right)=A\left(x^{2}-2 \alpha x+\left(\alpha^{2}+\beta^{2}\right)\right)
$$


where $z_{1}=\alpha+i \beta, \alpha, \beta \in \mathbb{R}, \beta \neq 0$. Since $P(0)>0$, the constant $A$ is positive. According to (9) and (7), the $q$-Bézier coefficient $p_{1}$ is defined by

$$
p_{1}=p(0,1 ; q)=A\left(\frac{-2 \alpha}{1+q}+\left(\alpha^{2}+\beta^{2}\right)\right)=A\left[\left|z_{1}-\frac{1}{1+q}\right|^{2}-\left(\frac{1}{1+q}\right)^{2}\right] .
$$

Accordingly, the assumption that $z_{1} \notin \mathbb{D}_{q}$ implies that $p_{1}>0$, and therefore $L_{q}(P)=2$.

We now assume that the two zeros $x_{1}^{*}, x_{2}^{*}$ belong to the interval $] 2 /(1+q), 1[$.

$$
P(x)=A\left(x-x_{1}^{*}\right)\left(x-x_{2}^{*}\right), \quad \text { with } A>0,
$$

the positivity of $A$ resulting again from $P(0)>0$. By (9) and (7), the second $q$-Bézier coefficient $p_{1}$ is given by

$$
p_{1}=A\left(x_{1}^{*} x_{2}^{*}-\frac{x_{1}^{*}+x_{2}^{*}}{1+q}\right)=r\left(x_{1}^{*}, x_{2}^{*}\right),
$$

where $r$ is the classical blossom of the quadratic polynomial $R$ defined by

$$
R(x)=x^{2}-\frac{2 x}{1+q}, \quad x \in \mathbb{R} .
$$

The classical Bézier coefficients $\left(r_{0}, r_{1}, r_{2}\right)$ of $R$ relative to $(2 /(1+q), 1)$ are

$$
r_{0}=R\left(\frac{2}{1+q}\right)=0, r_{1}=r\left(\frac{2}{1+q}, 1\right)=\frac{q-1}{(1+q)^{2}} \geq 0, r_{2}=R(1)=\frac{q-1}{1+q} \geq 0 .
$$

For any $u_{1}, u_{2} \in[2 /(1+q), 1]$, the real number $r\left(u_{1}, u_{2}\right)$ is a convex combination of the non-negative numbers $r_{0}, r_{1}, r_{2}$, and therefore $r\left(u_{1}, u_{2}\right) \geq 0$. In particular, from (34), we can assert that $p_{1} \geq 0$. This shows that $L_{q}(P)=2$, and the proof is complete.

Remark 6. Under the assumptions of Theorem 6, the polynomial $P$ satisfies $P(0)>0$ ), $P(1)>0$, and, as a consequence of Theorem $6, L_{q}(P)<+\infty$. In other words, $P$ satisfies conditon (ii) of Theorem 3. The equivalence (i) $\Leftrightarrow$ (ii) guarantees that $P$ is $q$-positive. This fact is actually visible at first sight: since the only possible real zeros of $P$ are located in the open interval $] 2 /(1+q), 1[, P$ is indeed positive on $[0,1] \backslash] 2 /(1+q), 1[$, and therefore on the whole lattice $\overline{\mathscr{A}}_{q}$ since $1 / q<2 /(1+q)$.

\section{Upper bounds for quantum Lorentz degrees}

Given a polynomial $P \in \mathbb{P}_{n}$ positive on $[0,1]$, with Bézier coefficients $p_{0}^{\star}, \ldots, p_{n}^{\star}$ relative to $(0,1)$, it is well known that its classical Lorentz degree admits the following upper bound, see [28]:

$$
L_{[0,1]}(P) \leq\left\lceil\left(\begin{array}{l}
n \\
2
\end{array}\right) \frac{M^{\star}}{\lambda^{\star}}\right\rceil,
$$

where $\lambda^{\star}$ is the minimum of $P$ on $[0,1], M^{\star}:=\max _{i=0}^{n}\left|p_{i}^{\star}\right|$, and where $\lceil$.$\rceil stands for$ the ceiling function. We want to establish a similar bound for the $q$-Lorentz degrees. Again, without loss of generality we can assume that $q>1$ is fixed. Indeed, for $q<1$, through (17) an upper bound for $L_{q^{-1}}(T(P))$ will provide an upper bound for $L_{q}(P)$. 


\subsection{A general upper bound}

To establish an upper bound for the quantum Lorenz degree of $q$-positive polynomials, we need the following lemma.

Lemma 4. For any non-negative integers $n, k, j, N$, such that $k \leq n \leq N$ and $0 \leq j \leq N$, and any $q>1$,

$$
\left(q^{-N} ; q\right)_{n} B_{k}^{n}\left(q^{j-N},\left(q^{-N}, 1\right) ; q\right) \leq B_{k}^{n}\left(q^{j-N},(0,1) ; q\right) .
$$

Proof. Observing that $\left(1-q^{-N}\right)_{q}^{n}=\left(q^{-N} ; q\right)_{n}$ and that, for all integers considered, we have $\left(1-q^{j-N}\right)_{q}^{n-k} \geq 0$, the inequalities (36) are direct consequences of the following ones:

$$
q^{(j-N) k} \geq\left(q^{j-N}-q^{-N}\right)_{q}^{k}
$$

Theorem 7. Given $q>1$, let $P$ be a q-positive polynomial of degree $n$, and let $p_{0}, p_{1}$, $\ldots, p_{n}$, be its $q$-Bézier coefficients relative to $(0,1)$. Then

$$
L_{q}(P) \leq\left\lceil\frac{\ln \left(M[n]_{q}\right)-\ln \lambda}{\ln q}\right\rceil
$$

where $\lambda$ is the infimum of $P$ on $\overline{\mathscr{A}_{q}}$ and $M:=\max _{i=0}^{n}\left|p_{i}\right|$.

Proof. The infimum $\lambda$ is a minimum and therefore $\lambda>0$. Set $\mathbf{p}:=\left(p_{0}, \ldots, p_{n}\right)$. For any $\omega \notin\left\{q^{-j} \mid j=0, \ldots, n-1\right\}$ and any $x \in \mathbb{R}$,

$$
(\omega ; q)_{n} \mathscr{P}(x ; \mathbf{p} ; \omega)=P(x)-\sum_{k=0}^{n} p_{k}\left[B_{k}^{n}(x,(0,1) ; q)-(\omega ; q)_{n} B_{k}^{n}(x,(\omega, 1) ; q)\right],
$$

where $\mathscr{P}(. ; \mathbf{p} ; \omega)$ is the function defined in (11). For $x \in \overline{\mathscr{A}_{q}}$ this implies

$$
(\omega ; q)_{n} \mathscr{P}(x ; \mathbf{p} ; \omega) \geq \lambda-M \sum_{k=0}^{n}\left|B_{k}^{n}(x,(0,1) ; q)-(\omega ; q)_{n} B_{k}^{n}(x,(\omega, 1) ; q)\right|
$$

In the previous relation, take $\omega=q^{-N}$ and $x=q^{j-N}$, for some $N \geq n$ and some nonnegative $j \leq N$. On account of Lemma 4 , we can then drop the absolute values within the sum, which then allows us to split the sum into two sums. This yields:

$$
\begin{array}{rl}
\left(q^{-N} ; q\right)_{n} & \mathscr{P}\left(q^{j-N} ; \mathbf{p} ; q^{-N}\right) \\
& \geq \lambda-M\left(\sum_{k=0}^{n} B_{k}^{n}\left(q^{j-N},(0,1) ; q\right)-\sum_{k=0}^{n}\left(q^{-N} ; q\right)_{n} B_{k}^{n}\left(q^{j-N},\left(q^{-N}, 1\right) ; q\right)\right) \\
& =\lambda-M+M\left(q^{-N} ; q\right)_{n} .
\end{array}
$$

Applying the inequality (see [28])

$$
\prod_{j=1}^{n}\left(1-x_{j}\right) \geq 1-\sum_{j=1}^{n} x_{j} \quad \text { for all } x_{1}, \ldots, x_{n} \in[0,1]
$$


we can see that $\left(q^{-N} ; q\right)_{n} \geq 1-[n]_{q} q^{-N}$. Accordingly,

$$
\lambda-M+M\left(q^{-N} ; q\right)_{n} \geq \lambda-M[n]_{q} q^{-N} .
$$

Take $N$ such that $q^{N} \geq M[n]_{q} / \lambda$, or equivalently, take $N \geq\left(\ln M[n]_{q}-\ln \lambda\right) / \ln q$. Then (38) shows that for any such value of $N$,

$$
\mathscr{P}\left(q^{j-N} ; \mathbf{p} ; q^{-N}\right) \geq 0, \quad 0 \leq j \leq N .
$$

Using Proposition 1, this proves (37).

In case $P$ is positive on $[0,1]$ we can improve the upper bound as follows:

Corollary 5. Let $P \in \mathbb{P}_{n}$ be positive on $[0,1]$. Then,

$$
L_{q}(P) \leq\left\lceil\min \left(\frac{\ln \left(M[n]_{q}\right)-\ln \lambda}{\ln q},\left(\begin{array}{l}
n \\
2
\end{array}\right) \frac{M^{\star}}{\lambda^{\star}}\right)\right\rceil,
$$

where $M^{\star}, \lambda^{\star}$ have the same meaning as in (35).

Observe that the numbers $M, \lambda$ depend on $q$, and that $\lim _{q \rightarrow 1^{+}} M=M^{\star}, \lim _{q \rightarrow 1^{+}} \lambda=$ $\lambda^{\star}, \lim _{q \rightarrow 1^{+}}[n]_{q}=n$. Accordingly,

$$
\lim _{q \rightarrow 1^{+}}\left(\frac{\ln \left(M[n]_{q}\right)-\ln \lambda}{\ln q}\right)=+\infty
$$

which means that, for $q$ sufficiently close to 1 , the upper bounds in (39) and (35) coincide.

\subsection{Improved upper bound for quadratic polynomials}

For the sake of simplicity we will focus on quadratic polynomials positive on the interval $[0,1]$. Taking account of Theorem 6 , we can establish a new upper bound, specific to quadratic polynomials.

Theorem 8. Let $P$ be a quadratic polynomial positive on $[0,1]$, with

$$
P(x)=x^{2}+a x+b, \quad x \in \mathbb{R} .
$$

If the zeros of $P$ are outside the disc $\mathbb{D}_{q}$, then $L_{q}(P)=2$. Otherwise

$$
L_{q}(P) \leq\left\lceil\frac{\ln (1+\xi(q-1))}{\ln q}\right\rceil
$$

where $\xi$ is the positive zero of the quadratic polynomial

$$
Q(x):=\left(4 b-a^{2}\right) x^{2}-(4 b-2 a(1+a)) x-(1+a)^{2} .
$$

Proof. That $L_{q}(P)=2$ when the zeros of $P$ are outside the disc $\mathbb{D}_{q}$ is a consequence of Theorem 6. Let us assume that $P$ has one zero inside $\mathbb{D}_{q}$. Since $P$ is positive in $[0,1]$, this zero is necessarily a non-real complex number, so $\Delta:=a^{2}-4 b<0$, and both zeros of $P$ are in $\mathbb{D}_{q}$. According to (7) and (8), the $q$-Bézier coefficients of $P$, viewed as a polynomial of degree $N$, are given by (see (9))

$$
p_{k}^{N}=\frac{\left[\begin{array}{l}
k \\
2
\end{array}\right]_{q}}{\left[\begin{array}{l}
N \\
2
\end{array}\right]_{q}}+a \frac{\left[\begin{array}{l}
k \\
1
\end{array}\right]_{q}}{\left[\begin{array}{l}
N \\
1
\end{array}\right]_{q}}+b, \quad k=0,1, \ldots, N .
$$


Thus

$$
[N]_{q}[N-1]_{q} p_{k}^{N}=[k]_{q}[k-1]_{q}+a[k]_{q}[N-1]_{q}+b[N]_{q}[N-1]_{q}, \quad k=0,1, \ldots, N .
$$

Using the fact that $[k-1]_{q}=q^{-1}\left([k]_{q}-1\right)$ for all $k \geq 1$, the previous relation can be written as:

$$
\begin{aligned}
q[N]_{q}[N-1]_{q} p_{k}^{N} & =[k]_{q}^{2}+\left(a[N]_{q}-a-1\right)[k]_{q}+b\left([N]_{q}^{2}-[N]_{q}\right), \\
& =f_{N}\left([k]_{q}\right), \quad k=0,1, \ldots, N,
\end{aligned}
$$

where $f_{N}$ denotes the quadratic function

$$
f_{N}(x)=x^{2}+\left(a[N]_{q}-a-1\right) x+b\left([N]_{q}^{2}-[N]_{q}\right), \quad x \in \mathbb{R} .
$$

The minimum of $f_{N}$ is reached at

$$
x_{N}=\frac{1+a-a[N]_{q}}{2},
$$

and this minimum is given by

$$
f_{N}\left(x_{N}\right)=\frac{1}{4} Q\left([N]_{q}\right),
$$

where the quadratic polynomial $Q$ is defined in (42). On account of (45), any integer $N \geq 2$ such that $Q\left([N]_{q}\right) \geq 0$ ensures that all $q$-Bézier coefficients $p_{k}^{N}, k=0, \ldots, N$, are non-negative. Now, the polynomial $Q$ has two real roots of opposite strict signs. Let $\xi$ denote the positive root, namely $\xi=2 b-a(1+a)+2 \sqrt{P(0) P(1)}$. We can thus state that

$$
[N]_{q} \geq \xi \quad \Rightarrow \quad p_{k}^{N} \geq 0 \text { for } k=0, \ldots, N .
$$

Now (41) follows from this observation.

\subsection{Illustration}

Consider the degree 2 polynomial $P$ given by

$$
P(x)=x^{2}-\frac{2}{3} x+\frac{1+\varepsilon}{9}, \quad \text { for some } \varepsilon>0 .
$$

The zeros of $P$ are the complex numbers

$$
z_{1}=\frac{1}{3}+i \frac{\sqrt{\varepsilon}}{3}, \quad z_{1}=\frac{1}{3}-i \frac{\sqrt{\varepsilon}}{3} .
$$

Thus $P$ is positive on the interval $[0,1]$. The $q$-Bézier coefficient of $P$ relative to $(0,1)$ are

$$
p_{0}=\frac{1+\varepsilon}{9}, \quad p_{1}=\frac{1+\varepsilon}{9}-\frac{2}{3+3 q}, \quad p_{2}=\frac{4+\varepsilon}{9} .
$$

Accordingly, for $q \geq(5-\varepsilon) /(1+\varepsilon)$, we have $L_{q}(P)=2$. In particular, independent of $\varepsilon, L_{q}(P)=2$ when $q \geq 5$. Note that this can also be viewed as an application of Theorem 6 since the real part of the roots $z_{1}, z_{2}$ satisfies $\frac{1}{3} \geq \frac{2}{q+1}$ if and only if $q \geq 5$. On the other hand, from

$$
\lambda^{\star}:=\min _{x \in[0,1]} P(x)=P\left(\frac{1}{3}\right)=\frac{\varepsilon}{9},
$$



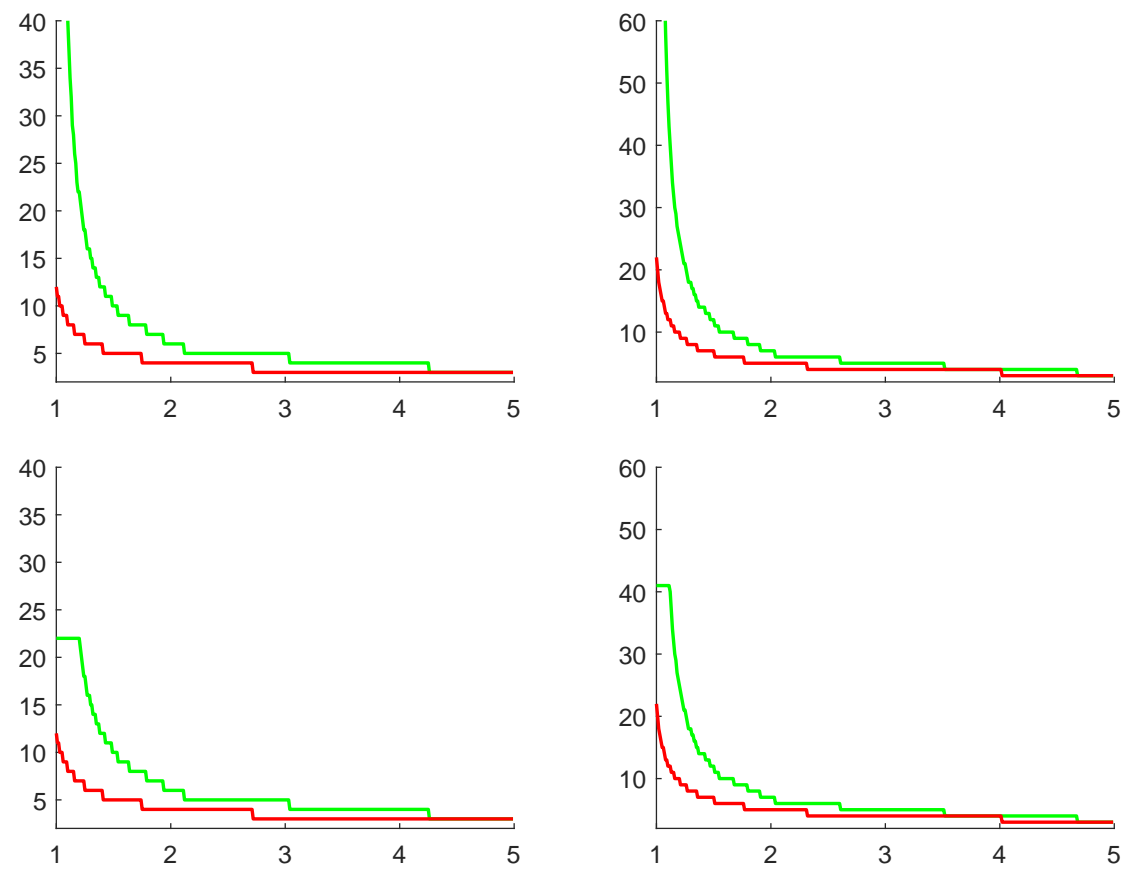

Figure 3: The various bounds for the $q$-Lorentz degree of the polynomial $P_{\varepsilon}$ defined in (47) as functions of $q \in] 1,5]$, with $\varepsilon=1 / 5$ (left), $\varepsilon=1 / 10$ (right). The red graphs correspond to (41). The green graphs illustrate (37) (top) and (39) (bottom).

we can derive that

$$
\lambda:=\min _{x \in \overline{\mathscr{A}}_{q}} P(x)=\min \left[P\left(\frac{1}{q^{j-1}}\right), P\left(\frac{1}{q^{j}}\right)\right] \quad \text { where } \quad j=\left\lceil\frac{\ln 3}{\ln q}\right\rceil .
$$

The various bounds obtained for the $q$-Lorentz degree of the poynomial $P$ are illustrated in Figure 2, as functions of $q \in] 1,5]$, for two different values of $\varepsilon, \varepsilon=1 / 5$ (left) and $\varepsilon=1 / 10$ (right). In each picture, the red graph shows the bound obtained in (41). As for the green graphs, they correspond to either (37) (top), or to the bound (39), with the exact value of $\lambda$ calculated via (49) (bottom).

\section{A quantum Pólya-type theorem}

The title of this article announced a generalisation of Pólya's theorem to polynomials positive on $q$-lattices, and this generalisation is the object of the present section.

The famous classical univariate Pólya theorem states that: given a polynomial $P$, positive on the interval $[0,+\infty[$, there exists an integer $M$ such that the coefficients of the polynomial

$$
Q(x)=(1+x)^{M} P(x)
$$

in the monomial basis are non-negative. The most natural extension of this result to the $q$-world might be stated as follows: given a polynomial $P$ positive on the $q$-lattice $\left\{q^{j} \mid j \in \mathbb{Z}\right\}$, with $q>1$, there exists a positive integer $M$ such that the coefficients of 
the polynomial

$$
(1+x)_{q}^{M} P(x)=q^{(M-1) M / 2} \prod_{i=0}^{M-1}\left(x+\frac{1}{q^{i}}\right) P(x)
$$

in the monomial basis are non-negative. This result would be true in particular for any polynomial $P$ positive on $] 0,+\infty[$. In other words, if such an extension were true, the infinite sequence $q^{-i}, j \geq 0$, would be Pólya positive in the sense of [3] or equivalently, the infinite sequence of degree one polynomials $\left(x+q^{-i}\right), j \geq 0$, would be strongly positive in the sense of [4]. This property is known to be true if and only if

$$
\sum_{i=0}^{+\infty} \min \left(q^{i}, \frac{1}{q^{i}}\right)=+\infty
$$

which is obviously false for $q \neq 1$.

This is why, prior to extending the Pólya theorem to the $q$-world, we have to rephrase it through the change of variable $y=1+x$. This yields the following statement: Given a polynomial $P$ of exact degree $n$, positive on the interval $[1,+\infty[$, there exists an integer $M$ such that the coefficients of the polynomial

$$
Q(y)=y^{M} P(y)
$$

in the basis $\left\{1,(y-1),(y-1)^{2}, \ldots,(y-1)^{M+n}\right\}$ are non-negative. This is the version of the Pólya theorem that we generalize to the $q$-world in Theorem 9 below.

Theorem 9. Fix $q>1$ and consider a polynomial $P$ of exact degree $n$ positive on the lattice $\mathscr{B}_{q}=\left\{q^{j}, j \in \mathbb{N}\right\}$. Then there exists a non-negative integer $M$ such that the coefficients in the basis

$$
\left\{1,(x-1)_{q}^{1},(x-1)_{q}^{2}, \ldots,(x-1)_{q}^{M+n}\right\}
$$

of the polynomial $Q_{M}(x)=x^{M} P(x)$ are non-negative.

The key-point in the proof of Theorem 9 is the following useful observation which can be derived in a straightforward manner by combining the definition of quantum Lorentz degrees with Proposition 2.

Proposition 4. Given a polynomial $P \in \mathbb{P}_{n}$, and any positive $q$, the following properties are equivalent:

(i) $L_{q}(P)<+\infty$;

(ii) there exists a non-negative integer $M$ such that all the coefficients of the polynomial $\Theta_{M+n}(P)$ in the basis (50) are non-negative.

Proof of Theorem 9: Since $P$ is positive on the lattice $\mathscr{B}_{q}$, the polynomial $\Theta_{n}(P)$ is positive on the lattice $\mathscr{A}_{q}$. Accordingly,

$$
\Theta_{n}(P)(0)=\lim _{j \rightarrow+\infty} \Theta_{n}(P)\left(q^{-j}\right) \geq 0 .
$$

Since the coefficients of $\Theta_{n}(P)$ in the monomial basis of degree $n$ are those of $P$ taken in the reverse order, $\Theta_{n}(P)(0)$ is the leading coefficient of $P$. Hence $\Theta_{n}(P)(0)$ is not zero since $P$ is of exact degree $n$. Therefore, $\Theta_{n}(P)(0)$ is positive, so the polynomial $\Theta_{n}(P)$ is positive on $\overline{\mathscr{A}}_{q}$, i.e., $\Theta_{n}(P)$ is $q$-positive. From Theorem 3 it follows that $L_{q}(P)<$ $+\infty$. Observing that for each non-negative integer $M$, the polynomial $\Theta_{M+n}\left(\Theta_{n}(P)\right)$ is the polynomial $Q_{M}$ introduced in Theorem 9, the claim follows from Proposition 4. 


\section{Quantum Lorentz degree: possible extension?}

As introduced in Definition 2, the notion of $q$-Lorentz degree is inherently connected with the $q$-degree elevation process on $[0,1]$. By contrast, the $q$-lattice which served to analyze the convergence is not intrinsically connected with $q$-degree elevation. Using this lattice is somehow contrary to the usual geometric design treatment of such corner cutting algorithms, whose convergence is generally addressed with the help of a non-nested grid adapted to it, that is, for each level $n \geq 1$, a sequence

$$
0=x_{n, 0}<x_{n, 1}<\cdots<x_{n, n-1}<x_{n, n}=1,
$$

from which the sequence of level $(n+1)$ results through the algorithm in question. This, for instance, is the approach for the classical degree elevation algorithm, where $x_{n, k}:=\frac{k}{n}, k=0, \ldots, n$, are the Greville points of degree $n$, i.e., the Bézier coefficients relative to $(0,1)$ of the identity $\Xi(x)=x, x \in \mathbb{R}$. More generally, the grids permitting the convergence analysis of any dimension elevation algorithms - the analogues of degree elevation concerning infinite nested sequences of Chebyshevian spaces - are formed by analogues of the Greville points, see [2]. Note that a similar principle applies to corner cutting subdivision schemes [20].

Here too one would naturally expect to use the $q$-Greville grid, that is, the nonnested grid formed by the $q$-Bézier coefficients of successive degrees of the identity $\Xi$ relative to $(0,1)$. According to (7), (9), and (8), for degree $n$, the $q$-Greville points provide a sequence of the form (51) defined by

$$
\xi_{n, k}=\frac{\sigma_{1}\left(1, q, \ldots, q^{k-1}\right)}{\sigma_{1}\left(1, q, \ldots, q^{n-1}\right)}=\frac{[k]_{q}}{[n]_{q}}, \quad k=0, \ldots, n .
$$

Surprisingly, we will see that this $q$-Greville grid appears naturally in an alternative analysis of the convergence of $q$-degree elevation on $[0,1]$ generated by embedding the quantum world in the mixed quantum-discrete world, which corresponds to embedding the class of all $q$-Bernstein bases in the larger class of all $(q, h)$-Bernstein bases. This embedding will also enable us to extend the notion of quantum Lorentz degree.

\section{1. ( $q, h)$-Bernstein bases and $(q, h)$-Lorentz degree}

Given any positive $q$ and any $h \in \mathbb{R}$, let the function $g$ be defined on $\mathbb{R}$ by $g(x)=$ $q x+h$, and for any non-negative integer $j$ let $g^{[j]}$ be defined by $g^{[j]}:=\underbrace{g \circ g \circ \cdots \circ g}_{j \text { times }}$, that is,

$$
g^{[j]}(x)=q^{j} x+[j]_{q} h, \quad x \in \mathbb{R}, \quad j \geq 0 .
$$

For any $a, b$ such that $b \neq g^{[j]}(a), j=0,1, \ldots, n-1$, consider the functions defined on $\mathbb{R}$ by

$$
B_{k}^{n}(x ;(a, b) ; q, h)=\left[\begin{array}{l}
n \\
k
\end{array}\right]_{q} \frac{\prod_{j=0}^{k-1}\left(x-g^{[j]}(a)\right) \prod_{j=0}^{n-k-1}\left(b-g^{[j]}(x)\right)}{\prod_{j=0}^{n-1}\left(b-g^{[j]}(a)\right)}, \quad k=0,1, \ldots, n
$$

These functions form a normalized basis for $\mathbb{P}_{n}$, called the $(q, h)$-Bernstein basis relative to $(a, b)$, see [15]. When a polynomial $P \in \mathbb{P}_{n}$ is expressed in this basis as

$$
P=\sum_{k=0}^{n} p_{k} B_{k}^{n}(.,(a, b) ; q, h)
$$


its $(q, h)$-Bézier coefficients $p_{k}$ relative to $(a, b)$ are given by

$$
p_{k}=p\left(g^{[k]}(a), \ldots, g^{[n-1]}(a), b, g(b), \ldots, g^{[k-1]}(b) ; q, h\right), \quad k=0, \ldots, n,
$$

where $p(. ; q, h)$ is the $(q, h)$-blossom of $P$ defined as follows, see [15]:

Definition 4. Given any $P \in \mathbb{P}_{n}$, any positive $q$, and any $h$, the $(q, h)$-blossom of $P$ is the unique multi-affine symmetric function $p(. ; q, h)$ in $n$ variables, such that

$$
p\left(x, g(x), g^{[2]}(x), \ldots, g^{[n-1]} x ; q, h\right)=P(x), \quad x \in \mathbb{R} .
$$

Note that the $q$-Bernstein basis relative to $(a, b)$ is the $(q, 0)$-Bernstein basis relative to $(a, b)$, and for any polynomial $P \in \mathbb{P}_{n}$ the $q$-blossom of $P$ coincides with its $(q, 0)$ blossom.

When $b$ additionally satisfies $b \neq g^{[n]}(a)$, expanding a given polynomial $P \in \mathbb{P}_{n}$ in the corresponding $(q, h)$-Bernstein bases of degrees $n$ and $(n+1)$ relative to $(a, b)$, that is,

$$
P=\sum_{k=0}^{n} p_{k} B_{k}^{n}(.,(a, b) ; q, h)=\sum_{k=0}^{n} r_{k} B_{k}^{n+1}(.,(a, b) ; q, h),
$$

the new $(q, h)$-Bézier coefficients $r_{0}, \ldots, r_{n+1}$ can be computed from the initial ones $p_{0}, \ldots, p_{n}$, via $r_{0}=p_{0}, r_{n+1}=r_{n}$, and [14]:

$$
r_{i}=\frac{[n+1-i]_{q}}{[n+1]_{q}} p_{i}+q^{n+1-i} \frac{[i]_{q}}{[n+1]_{q}} p_{i-1}, \quad i=1, \ldots, n .
$$

Supposing that $b \neq g^{[j]}(a)$ for all $j \geq 0$, we can more generally expand $P$ as

$$
P=\sum_{k=0}^{n} p_{k}^{N} B_{k}^{N}(.,(a, b) ; q, h) \quad \text { for all } N \geq n,
$$

which enables us to introduce an associated Lorentz degree:

Definition 5. For any positive $q$, any real number $h$, and any $(a, b) \in \mathbb{R}^{2}$ such that $b \neq g^{[j]}(a)$ for all $j \geq 0$, the $(q, h)$-Lorentz degree of $P$, denoted by $L_{(q, h)}^{(a, b)}(P)$, is defined as the (possibly infinite) quantity

$$
L_{(q, h)}^{(a, b)}(P):=\inf \left\{N \geq n \mid p_{k}^{N} \geq 0 \text { for } k=0, \ldots, N\right\} .
$$

Remark 7. We conclude this subsection with some interesting remarks.

1- Since relation (55) is independent of $a, b, h$, it coincides with the $q$-degree elevation formula (10). Accordingly, formula (25) also describes the $(q, h)$-degree elevation from $\mathbb{P}_{n}$ to $\mathbb{P}_{N}$ relative to any $(a, b)$ such that $b \neq g^{[j]}(a), j=0,1, \ldots, N-1$.

2- Because formula (55) does not depend on $a, b, h$, the $(q, h)$-Lorentz degree $L_{(q, h)}^{(a, b)}(P)$ of a polynomial $P \in \mathbb{P}_{n}$ is not really attached to the polynomial $P$ : it depends only on $q$ and on the sequence $\left(p_{0}, \ldots, p_{n}\right)$ formed by the initial $(q, h)$-Bézier points of $P$ relative to $(a, b)$. We can thus introduce the $q$-Lorentz degree of the sequence $\left(p_{0}, \ldots, p_{n}\right)$ defined as

$$
\mathbf{L}_{q}\left(p_{0}, \ldots, p_{n}\right):=L_{(q, h)}^{(a, b)}(P)
$$


where the polynomial $P$ (depending on $q, h, a, b$ ) is given by (53) for any admissible $h, a, b$, that is, $b \neq g^{[j]}(a)$ for all $j \geq 0$. Of course, in particular we have

$$
\mathbf{L}_{q}\left(p_{0}, \ldots, p_{n}\right):=L_{q}(P), \quad \text { where } P=\sum_{k=0}^{n} p_{k} B_{k}^{n}(.,(0,1) ; q) .
$$

Accordingly, the properties of the $q$-Lorentz degree developed in the previous sections can now be stated in terms of $\mathbf{L}_{q}$. To cite only one elementary example, relation (17) can be replaced by

$$
\mathbf{L}_{q}\left(p_{0}, \ldots, p_{n}\right)=\mathbf{L}_{q^{-1}}\left(p_{n}, \ldots, p_{0}\right) \text { for each positive } q \text { and each }\left(p_{0}, \ldots, p_{n}\right) \in \mathbb{R}^{n} .
$$

Then whenever $a, b, h$ and $\bar{a}, \bar{b}, \bar{h}$ satisfy the standard conditions, this formula can in turn be written as

$$
L_{(q, h)}^{(a, b)}(P)=L_{\left(q^{-1}, \bar{h}\right)}^{(\bar{a}, \bar{b})}(Q),
$$

where $P$ is defined in (53) and $Q:=\sum_{k=0}^{n} p_{n-k} B_{k}^{n}\left(\cdot,(\bar{a}, \bar{b}) ; q^{-1}, \bar{h}\right)$. These comments explain why it is not really worthwhile investigating more deeply the notion of $(q, h)$ Lorentz degrees.

3- Again due to the fact that $q$-degree elevation depends only on $q$, whatever (admissible) interval $[a, b]$ we are working on, the limit of a given initial $q$-control polygon relative to $(a, b)$ under the infinite degree elevation process is the same: this limit is the piecewise linear polygon whose infinitely many vertices are all located either on the $q$ Bézier curve defined on $[0,1]$ by the initial polygon $(q>1)$ or on the $q^{-1}$-Bézier curve defined on $[0,1]$ by the reverse initial polygon $(q<1)$. However when working on another interval $[a, b]$, the position of the limit vertices is no longer connected with the $q$ - or $q^{-1}$-Bézier curve defined on the interval $[a, b]$ by the initial polygon or its reverse. This phenomenon is clearly illustrated in Figure 4, left, where we compare the degree $N=100$ elevated polygon (red polygon) with the $q$-Bézier curve on $[a, b]$ with $q=1.4$. For $q=0.5$ (right), we show both the $q$-curve and the $q^{-1}$-curve with reverse initial control polygon on $[a, b]$, at least a reasonable part of this curve, for this curve goes very far from the initial polygon. In both cases, the blue curve is the above-mentioned $q$ - or $q^{-1}$-Bézier curve on $[0,1]$ on which the limit vertices are located.

4-The same comments are valid for $(q, h)$ curves when $h \neq 0$, even on the interval $[0,1]$ as illustrated in Figure 5 with $(q, h)=(1.4,0.5)$ (left) and $(q, h)=(2,-2)$ (right). In the left picture we deliberately show the entire green $(q, h)$-Bézier curve for comparison with the right picture in order to show the joint effects of the parameters $q, h$ as investigated in [14].

\subsection{Convergence of $q$-degree elevation and $q$-Greville points}

This subsection presents an alternative approach to the convergence of the $q$-degree elevation algorithm already addressed in Section 3.

We start with the following $(q, h)$-counterpart of Lemma 1. We assume that the positive number $q$ is fixed.

Lemma 5. For any non-negative $n$, any $a, h \in \mathbb{R}$, with $h \neq(1-q) a$, the expansion of any polynomial $P \in \mathbb{P}_{n}$ in the $(q, h)$-Bernstein basis relative to $\left(a, g^{[n]}(a)\right)$ is

$$
P=\sum_{k=0}^{n} P\left(g^{[k]}(a)\right) B_{k}^{n}\left(.,\left(a, g^{[n]}(a)\right) ; q, h\right) .
$$



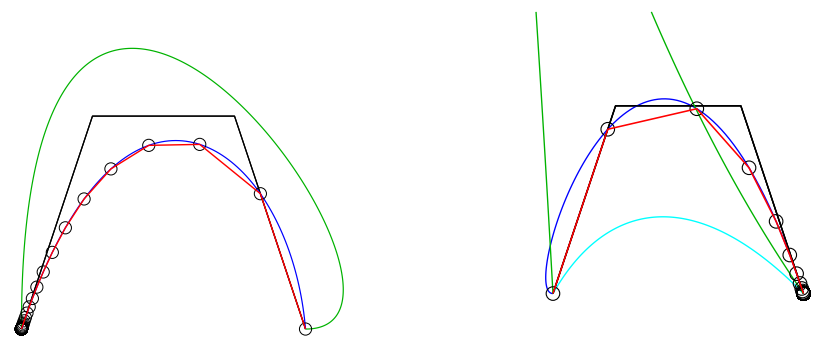

Figure 4: Comparison between the (red) $q$-degree elevated limit of the initial (black) $q$-control polygon and the quantum curves on $[3,7]$ generated by either the black polygon or its reverse. Left: $q=1.4$. Right: $q=0.5$. See Remark 7, item 3 .
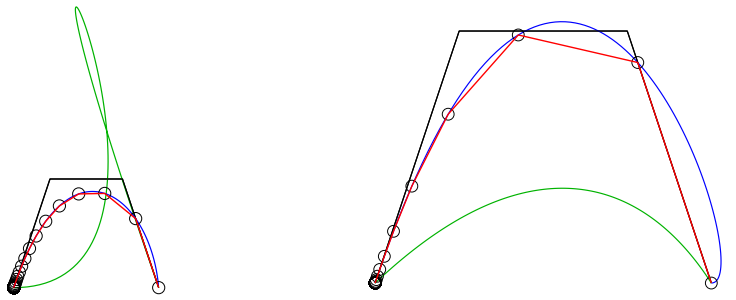

Figure 5: Comparison between the (red) $(q, h)$-degree elevated limit of the initial (black) $(q, h)$-control polygon and the corresponding cubic $(q, h)$-curve on $[0,1]$, with $(q, h)=(1.4,0.5)($ left $) ;(q, h)=(2,-2)$ (right).

Proof. The requirement $h \neq(1-q) a$ guarantees that the $(q, h)$-Bernstein basis relative to $\left(a, g^{[n]}(a)\right)$ is meaningful. Setting $\alpha:=g^{[k]}(a)$, we can see that

$$
\left(g^{[k]}(a), \ldots, g^{[n-1]}(a), b, g(b), \ldots, g^{[k-1]}(b)\right)=\left(\alpha, g(\alpha), \ldots, g^{[n-1]}(\alpha)\right) .
$$

Therefore by (54) and Definition 4, relative to $\left(a, g^{[n]}(a)\right)$ the $(q, h)$-Bézier coefficient of $P$ of index $k$ is equal to $P(\alpha)$.

Remark 8. Under the same assumptions as in Lemma 5, the expansion (57) means that the $(q, h)$-Bernstein basis relative to $\left(a, g^{[n]}(a)\right)$ is the Lagrange basis associated with the sequence $\left(a, g(a), g^{[2]}(a), \ldots, g^{[n]}(a)\right)$; see [14, Section 4, Item 3]. As a special case we recover the degree $n q$-Greville points (52). Indeed,

$$
\text { for } a=0 \text { and } h=\frac{1}{[n]_{q}}, \quad g^{[k]}(a)=\frac{[k]_{q}}{[n]_{q}}=\xi_{n, k}, \quad k=0, \ldots, n \text {. }
$$

With any sequence $\mathbf{p}:=\left(p_{0}, \ldots, p_{n}\right)$ of real numbers, let us associate the following family of polynomials with parameter $h$ by setting:

$$
\mathscr{L}(. ; \mathbf{p} ; h)=\sum_{k=0}^{n} p_{k} B_{k}^{n}(.,(0,1) ; q, h), \quad h \notin\left\{1 /[j]_{q} \mid j=1, \ldots, n-1\right\} .
$$

Applying degree elevation to this family, we can state:

Proposition 5. For each $\mathbf{p}:=\left(p_{0}, \ldots, p_{n}\right)$, and each $h \notin\left\{1 /[j]_{q} \mid j=1, \ldots, N-1\right\}$,

$$
\mathscr{L}(. ; \mathbf{p} ; h)=\sum_{k=0}^{N} \mathscr{L}\left(\frac{[k]_{q}}{[N]_{q}} ; \mathbf{p} ; \frac{1}{[N]_{q}}\right) B_{k}^{N}(.,(0,1) ; q, h), \quad \text { for all } N \geq n .
$$



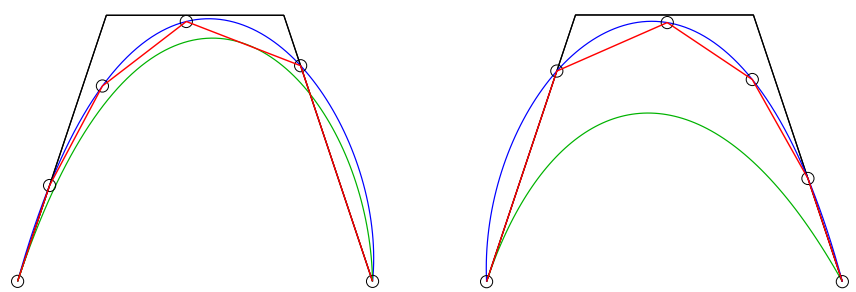

Figure 6: Initial (black) $q$-control polygon elevated to degree $N=5$ (red polygon), with the green cubic $q$-Bézier curve and the blue cubic $\left(q, \frac{1}{[N]_{q}}\right)$-Bézier curve it generates on $[0,1]$. Left: $q=1.5$. Right: $q=0.7$.

Proof. The proof is similar to that of Proposition 1. It is based on Lemma 5, on (58), and on the fact that the $(q, h)$-degree elevation formula (55) depends only on $q$.

Take any $N \geq n$, and any admissible up to some degree $N \geq n, a, b, h$ such that $b \neq$ $g^{[j]}(a), j=0,1, \ldots, N-1$. Then, with $\mathbf{p}=\left(p_{0}, \ldots, p_{n}\right)$, we associate the polynomial $P$ expanded as

$$
P=\sum_{k=0}^{n} p_{k} B_{k}^{n}(.,(a, b) ; q, h)=\sum_{k=0}^{N} p_{k}^{N} B_{k}^{N}(.,(a, b) ; q, h) \text { for } N .
$$

From the comments in the previous subsection, along with Propositions 1 and 5, we know that the $q$-Bézier coefficients $p_{k}^{N}$ satisfy

$$
p_{k}^{N}=\mathscr{P}\left(q^{k-N} ; \mathbf{p} ; q^{-N}\right)=\mathscr{L}\left(\frac{[k]_{q}}{[N]_{q}} ; \mathbf{p} ; \frac{1}{[N]_{q}}\right), \quad k=0, \ldots, N .
$$

Apply $(61)$ with $(a, b)=(0,1)$ and $h=0$. In that case, $P=\mathscr{P}(. ; \mathbf{p} ; 0)=\mathscr{L}(. ; \mathbf{p} ; 0)$. Let us focus on the right-hand side of (62). It says that after $(N-n)$ degree elevation steps, the vertices of the final $q$-control polygon are located on the $\left(q, \frac{1}{[N]_{q}}\right)$-Bézier curve generated on $[0,1]$ by the initial polygon. With $n=3$ and $N=5$, this feature is illustrated in Figure 6, with $q=1.5$ (left) and $q=0.7$ (right). Note that this phenomenon could as well be illustrated after replacing $q$ by $q^{-1}$ and the initial polygon by its reverse. Furthermore, the right-hand side of (62) also enables us to prove Theorem 10 below via arguments similar to those used for Theorem 1 , with $q^{j-N}$ replaced by $\frac{[j]_{q}}{[N]_{q}}$ and the uniform convergence on $[0,1]$ of the function $\mathscr{P}(. ; \mathbf{p} ; \omega)$ to $P$ when $\omega \rightarrow 0$ by the uniform convergence on $[0,1]$ of the function $\mathscr{L}(. ; \mathbf{p} ; h)$ to $P$ when $h \rightarrow 0$. Last but not least, when $q>1, \lim _{N \rightarrow+\infty} 1 /[N]_{q}=0$. Details are left to the reader.

Theorem 10. Suppose that $q>1$. Let $P \in \mathbb{P}_{n}$ be expanded in the $q$-Bernstein basis relative to $(0,1)$ for any degree $N \geq n$ as $P=\sum_{k=0}^{N} p_{k}^{N} B_{k}^{N}(.,(0,1) ; q)$. Then,

$$
\lim _{N \rightarrow+\infty} \max _{j=0,1, \ldots, N}\left|P\left(\frac{[j]_{q}}{[N]_{q}}\right)-p_{j}^{N}\right|=0
$$

For each positive integer $m$, we introduce the set

$$
\mathscr{C}_{q}^{m}:=\bigcup_{N=m}^{\infty}\left\{\frac{[j]_{q}}{[N]_{q}}, j=0,1, \ldots, N\right\} .
$$

We say that a polynomial $P \in \mathbb{P}_{n}$ is " $q$-positive" $(q>1)$ if there exists an integer $m \geq n$ such that $P$ is positive on the compact closure $\overline{\mathscr{C}_{q}^{m}}$. If so, since the infimum of $P$ on $\overline{\mathscr{C}_{q}^{m}}$ is positive, Theorem 10 enables us to state: 
Corollary 6. If $q>1$ and if $P \in \mathbb{P}_{n}$ is "q-positive", the $q$-Lorentz degree of $P$ is finite.

Defined in terms of the natural grid associated with the $q$-degree elevation algorithm, " $q$-positivity" seems to be the natural sufficient condition ensuring that the $q$ Lorentz degree is finite. Let us now compare this " $q$-positivity" with the previous notion of $q$-positivity introduced in Definition 3 for $q>1$. First observe that for each $j \geq 0$,

$$
\frac{[N-j]_{q}}{[N]_{q}}=q^{-j} \frac{1-q^{j-N}}{1-q^{-N}} \quad \text { for all } N \geq j .
$$

It readily follows that $\mathscr{A}_{q} \subset \overline{\mathscr{C}_{q}^{m}}$ for each $m \geq 1$. Accordingly, $q$-positivity is a weaker sufficient condition than " $q$-positivity" for providing a finite $q$-Lorentz degree. The additional greater difficulty to handle the sets $\overline{\mathscr{C}_{q}^{m}}, m \geq 1$, by comparison with $\overline{\mathscr{A}}_{q}$ highly justifies the somewhat artificial use of the $q$-lattice $\overline{\mathscr{A}_{q}}$ for the convergence analysis of $q$-degree elevation. Besides, $q$-lattices are also more standard tools in different mathematical areas, such as the theory of $q$-orthogonal polynomials [18, 32].

Without entering into all the technical details, we now briefly describe the $(q, h)$ approach when $q<1$. Let us call the reader's attention to the following major difference with the previous case:

$$
\text { if } q<1, \text { then } \lim _{N \rightarrow+\infty} \frac{1}{[N]_{q}}=1-q .
$$

For this reason, we will have to consider the uniform convergence on $[0,1]$ of $\mathscr{L}(. ; \mathbf{p} ; h)$ when $h \rightarrow 1-q$ rather than $h \rightarrow 0$. This leads to the following result:

Theorem 11. Suppose that $q<1$. Let $P \in \mathbb{P}_{n}$ be expanded in the $q$-Bernstein basis relative to $(0,1)$ for any degree $N \geq n$ as $P=\sum_{k=0}^{N} p_{k}^{N} B_{k}^{N}(.,(0,1) ; q)$. Then,

$$
\lim _{N \rightarrow+\infty} \max _{j=0,1, \ldots, N}\left|T_{q}(P)\left(\frac{[N-j]_{q^{-1}}}{[N]_{q^{-1}}}\right)-p_{j}^{N}\right|=0 .
$$

Proof. The proof involves two simple relations between $q$ - and $q^{-1}$-binomial coefficients, valid for any positive $q$ and any positive integer $n$ :

$$
\left[\begin{array}{l}
n \\
k
\end{array}\right]_{q}=q^{k(n-k)}\left[\begin{array}{l}
n \\
k
\end{array}\right]_{q^{-1}}, \quad 1-\frac{[k]_{q}}{[N]_{q}}=\frac{[N-k]_{q^{-1}}}{[N]_{q^{-1}}}, \quad k=0, \ldots, n .
$$

Recall from Subsection 3.3 that the polynomial $T_{q}(P)$ satisfies

$$
T_{q}(P)=\sum_{k=0}^{N} p_{N-k}^{N} B_{k}^{N}\left(.,(0,1) ; q^{-1}\right) \quad \text { for each } N \geq n .
$$

For $0 \leq k \leq n$, we have

$$
B_{k}^{n}(x,(0,1) ; q, 1-q)=B_{n-k}^{n}\left(1-x,(0,1) ; q^{-1}\right), \quad x \in \mathbb{R} .
$$

We skip the technical details leading to (67), simply mentioning that the proof uses the first relation in (66). We now assume that $q<1$. It is easily derived from (67) and (64) that, when $N \rightarrow+\infty, \mathscr{L}\left(. ; \mathbf{p} ; \frac{1}{[N]_{q}}\right)$ converges to $T_{q}(P)(1-x)$, uniformly on $[0,1]$. This convergence enables to conclude that

$$
\lim _{N \rightarrow+\infty} \max _{j=0,1, \ldots, N}\left|T_{q}(P)\left(1-\frac{[j]_{q}}{[N]_{q}}\right)-p_{j}^{N}\right|=0,
$$

which is the same as (65) due to the second formula in (66). 
As a by-product, (67) provides us with the following identity, valid for any $\mathbf{p}$ :

$$
T_{q}(\mathscr{P}(. ; \mathbf{p} ; 0))(x)=\mathscr{L}(1-x ; \mathbf{p} ; 1-q), \quad x \in \mathbb{R} .
$$

Let us conclude with an obvious consequence of Theorem 11 which could be followed by comments similar to those following Corollary 6 .

Corollary 7. If $q<1$ and if $P \in \mathbb{P}_{n}$ is "q-positive" in the sense that $T_{q}(P)$ is " $q^{-1}$ positive”, the q-Lorentz degree of $P$ is finite.

\section{Concluding comments}

With each polynomial $P$ we can associate infinitely many (possibly infinite) Lorentz degrees on the interval $[0,1]$, namely, in addition to its classical Lorentz degree $L_{[0,1]}(P)$, each quantum Lorentz degree $L_{q}(P), q>0, q \neq 1$. The convergence analysis of iterated $q$-degree elevation enabled us to provide a sufficient condition ( $q$-positivity) for $L_{q}(P)$ to be finite, which is a natural generalization of Bernstein's theorem. We provided new expressions for the $q$-Bézier coefficients of a polynomial $P$ in terms of divided differences, which was essential both to establish various properties of $q$-Lorentz degrees, and to derive a $q$-version of the univariate Pólya theorem for $q>1$. The notion of $q$ Lorentz degree can be extended to any admissible interval $[a, b]$, even within the larger mixed quantum-discrete framework. Still, the key point remains $q$-degree elevation on $[0,1]$ to which we have to return either to analyze the convergence on such an interval $[a, b]$ or to derive properties of the corresponding $q$ - or $(q, h)$-Lorentz degrees.

The final word about this work could be: the quantum realm never fails to be puzzling. For instance, whatever the positive number $q \neq 1$, the $q$-degree elevation algorithm looks like a perfect shape preserving geometric design algorithm which additionally is independent of the interval. Still, for a fixed $q$-control polygon, the resulting $q$-Bézier curve does depend on the interval. Moreover, the case $q>1$ is known to be of no interest for geometric design, for the initial $q$-control polygon hardly controls anything about the shape of the resulting curve which is not even located in its convex hull. This forces the curve to somehow perform contortions, for it has anyway to pass through all the vertices of the limit of the sequence of control polygons located inside the convex hull in question. This phenomenon can already be guessed for $q=2.4$ in Figure 1, and the phenomenon gets worse and worse as $q$ increases. Intuitively, we would expect the behaviour of the shape preserving case $q<1$ to be more consistent with the classical case $q=1$ relative to $q$-degree elevation, but it is not at all so. Indeed, as visible in Figure 2, when $q<1$ the limit vertices no longer lie in the proximity of the $q$-Bézier curve. The puzzling feature of the quantum world thus transpires: whenever we want to find the $q$-version of a classical property, it is impossible to easily predict if it will work for $q<1$, or $q>1$, or both, or none. To cite only one more example, finite $q$-Lorentz degrees convey some positivity preservation, and it is therefore surprising that the non-shape preserving case $q>1$ achieves better results than the optimal shape preserving case $q=1$, see (33).

Future work will concern quantum Lorentz degree dependent Markov inequalities in the spirit of [30].

\section{References}

\section{References}

[1] R. Ait-Haddou, On the Lorentz degree of a product of polynomials. J. Approx. Theory, 189 (2015), 81-87. 
[2] R. Ait-Haddou and M.-L. Mazure, Approximation by Chebyshevian Bernstein Operators versus Convergence of Dimension Elevation, Constr. Approx., 43 (2016), 425-461.

[3] R. Ait-Haddou, M.-L. Mazure, H. Render, Nested sequences of rational spaces: Bernstein approximation, dimension elevation, and Pólya-type theorems on positive polynomials, J. Approx. Theory, 234 (2018), 20-50.

[4] B. M. Baker, D. E. Handelman, Positive polynomials and time dependent integer-valued random variables, Can. J. Math., 44 (1992), 3-41.

[5] S.N. Bernstein, Sur la représentation des polynômes positifs, Collected Papers of S. N. Bernstein, Vol. 1, Constructive Theory of Functions (1905 to 1930), Academy of Sciences, USSR (1952), 251-252 [(in Russian); the original paper was published in 1915].

[6] J.-M. Carnicer, J.-M. Peña, Shape preserving representations and optimality of the Bernstein basis, Adv. Comput. Math., 1 (1993), 173-196.

[7] C. de Boor, Divided differences, Surveys Approx. Theory, 1 (2005), 466-9.

[8] G. Chang, J. Hoschek, Convergence of Bézier triangular nets and a theorem of Pólya, J. Approx. Theory, 58 (1989), 247-258

[9] T. Erdélyi, Estimates for the Lorentz degree of polynomials, J. Approx. Theory, 67 (1991), 187-198.

[10] T. Erdélyi, George Lorentz and inequalities in approximation, St. Petersburg Math. J., 21 (2010), 365-405.

[11] T. Erdélyi, J. Szabados, On polynomials with positive coefficients, J. Approx. Theory, 54 (1988), 107-122.

[12] T. Erdélyi, Inequalities for Lorentz polynomials, J. Approx. Theory, 192 (2015), 297-305.

[13] R. Goldman, Pyramid algorithms: A dynamic programming approach to curves and surfaces for geometric modeling. Elsevier, 2002.

[14] R. Goldman, P. Simeonov, Two essential properties of $(q, h)$-Bernstein-Bézier curves, Applied Numerical Mathematics, 96 (2015), 82-93.

[15] R. Goldman, P. Simeonov, Quantum Bernstein bases and quantum Bézier curves, J. Comp. Appl. Math., 288 (2015), 284-303.

[16] T.N.T. Goodman, H. Oruç, G.M. Phillips, Convexity and Generalized Bernstein polynomials, Proc. Edinburgh Mth. Soc., 42 (1999), 179-190.

[17] V. Kac, P. Cheung, Quantum calculus, Universitext series, IX, Springer Verlag, 2002.

[18] R. Koekoek, P.A. Lesky, R.F. Swarttouw, Hypergeometric Orthogonal Polynomials and their $q$-Analogues. Springer Monographs in Mathematics. Springer, Berlin (2010).

[19] M.-L. Mazure, Blossoms and optimal bases, Adv. Comp. Math., 20 (2004), 177-203.

[20] M.-L. Mazure, Subdivision schemes and non nested grids, Trends and Applications in Constructive Approximation, International Series of Numerical Mathematics, 151, D.H. Mache, J. Szabados, M.G. de Bruin (eds), 135-163, Birkhäuser, 2005.

[21] C.A. Micchelli, A. Pinkus, Some remarks on nonnegative polynomials on polyhedra, T.W. Anderson, et al. (Eds.), Probability, Statistics and Mathematics: Papers in Honor of Samuel Karlin, Ac. Press, Boston (1989), 163-186. 
[22] H. Oruç, G.M. Phillips, $q$-Bernstein polynomials and Bézier curves, J. Comp. Appl. Math, 151 (2003), 1-12.

[23] G.M. Phillips, On generalized Bernstein polynomials, AR Mitchell 75th Birthday Volume, World Scientific, 1996, 263-269.

[24] G.M. Phillips, A de Casteljau algorithm for generalized Bernstein polynomials, BIT Numerical Mathematics, 37 (1997), 232-236.

[25] G.M. Phillips, A survey of results on the $q$-Bernstein polynomials, IMA J. Num. Analysis, 30 (2010), 277-288.

[26] G. Pólya, Uber positive Darstellung von Polynomen Vierteljahrsschr. Naturforsch. Ges. Zuer., 73 (1928), 141-145.

[27] G. Pólya, G. Szegö, Problems and theorems in analysis, Volume II, p. 78, Problem 49, Springer Verl. Berlin, 1976.

[28] V. Powers, B. Reznick, A new bound for Pólyas theorem with applications to polynomials positive on polyhedra, J. Pure Appl. Algebra, 164 (2001), 221-229.

[29] H. Prautzsch, K. Kobbelt, Convergence of subdivision and degree elevation, Adv. Comput. Math., 2 (1994), 143-154.

[30] J.T. Scheick, Inequalities for derivatives of polynomials of special type, J. Approx. Theory, 6 (1972), 354-358.

[31] P. Simeonov, V. Zafiris, R. Goldman, $q$-Blossoming: A new approach to algorithms and identities for $q$-Bernstein bases and $q$-Bézier curves, J. Approx. Theory 164 (2012), 77-104.

[32] W. Van Assche, Q. Van Baelen, Zero Distribution of Orthogonal Polynomials on a $q$ Lattice, Constr. Approx. (2020). https://doi.org/10.1007/s00365-020-09513-2 